\title{
TANGENTIAL STIFFNESS OF ELASTIC MATERIALS WITH SYSTEMS OF GROWING OR CLOSING CRACKS
}

\author{
PERE C. PRAT $\dagger$ and ZDENĚK P. BAŽANT $\ddagger$ \\ †Department of Civil Engineering, Technical University of Catalunya, E-08034 Barcelona, Spain;

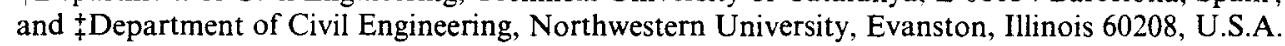

(Received 9 February 1996; in revised form 4 July 1996)

\begin{abstract}
Although much has been learned about the elastic properties of solids with cracks, virtually all the work has been confined to the case when the cracks are stationary, that is, neither grow nor shorten during loading. In that case, the elastic moduli obtained are the secant moduli. The paper deals with the practically much more important but more difficult case of tangential moduli for incremental deformations of the material during which the cracks grow while remaining critical, or shorten. Several families of cracks of either uniform or random orientation, characterized by the crack density tensor, are considered. To simplify the solution, the condition of crack criticality, i.e. the equality of the energy release rate to the energy dissipation rate based on the fracture energy of the material, is imposed only globally for all the cracks in each family, rather than individually for each crack. Sayers and Kachanov's approximation of the elastic potential as a tensor polynomial that is quadratic in the macroscopic stress tensor and linear in the crack density tensor, with coefficients that are general nonlinear functions of the first invariant of the crack density tensor, is used. The values of these coefficients can be obtained by one of the well-known schemes for elastic moduli of composite materials, among which the differential scheme is found to give more realistic results for post-peak strain softening of the material than the self-consistent scheme. For a prescribed strain tensor increment, a system of $N+6$ linear equations for the increments of the stress tensor and of the crack size for each of $N$ crack families is derived. Iterations of each loading step are needed to determine whether the cracks in each family grow, shorten, or remain stationary. The computational results are qualitatively in good agreement with the stress-strain curves observed in the testing of concrete. (C) 1997 Elsevier Science Ltd. All rights reserved
\end{abstract}

Keywords: A. microcracking, B. concrete, B. constitutive behaviour, B. crack mechanics, C. rock.

\section{INTRODUCTION}

A system of cracks (or microcracks) in a solid affects its stiffness, strength and ductility. Evolution of the cracks is a mechanism of failure. In rock mechanics, careful attention must be paid to the families of cracks, preexisting or man-made, which can have a strong influence on the response of the rock during excavations, tunneling, drilling and mining. In the fabrication of ceramics and composites, introduction of a predetermined system of microcracks can be used to control the effective stiffness of the material. In concrete, the growth of microcracks caused by loading has long been recognized as a major influence on the response of the structure.

Prediction of the response of structures made of damaging materials such as rock, 
concrete, ice, ceramics or composites requires modeling of the effect of microcracks on the macroscopic constitutive law. The basic problem is the effect of a crack system on the elastic constants of the material. The distribution pattern of the cracks within the body may vary from highly random and statistically uniform patterns which render the body macroscopically isotropic, to aligned patterns with some preferred crack orientations which render the body macroscopically anisotropic. The crack density can also vary from dilute (non-interacting) cracks to highly concentrated (heavily interacting) cracks, depending on the type of material, fabrication process, geological history and loading conditions.

The prediction of the effective elastic properties of microcracked solids is a classical problem of micromechanics of materials, which has received considerable attention since the mid-sixties. Two approaches have been used. The first focused on precise modeling of microcrack opening, propagation and interactions (Kachanov, 1987a; Horii and Nemat-Nasser, 1985), and the second focused on modeling of the overall effective elastic properties of a microcracked body by taking into account the crack opening and interactions only approximately.

One effective method in the latter, global micromechanics approach is the selfconsistent scheme. This scheme can be traced back in solid mechanics to the studies of Hill (1965) and Budiansky (1965) on the overall elastic moduli of multiphase materials, which extended previous pioneering studies of Hershey and Dahlgren (1954), Eshelby (1957) and Kröner (1958). Further refinements of this method include the three-phase generalized self-consistent method (Christensen and Lo, 1979). Other methods, such as the differential scheme (Roscoe, 1952 ; Salganik, 1973; Zimmerman, 1985; Hashin, 1988), the Mori-Tanaka method (Mori and Tanaka, 1973), and Dvorak's recent method of uniform fields (Dvorak and Benveniste, 1992; Dvorak, 1992), have also been proven useful. Using these techniques, the effect of various types of microcrack systems on the overall elastic properties of a body has been studied extensively, and many important results have been achieved in fields ranging from composite manufacture to geophysical research, rock mechanics and high-performance concretes (Budiansky and O'Connell, 1976; Hoenig, 1978, 1979; Kachanov, 1980; Horii and Nemat-Nasser, 1983; Fabrikant, 1987; Ju and Tseng, 1992 ; Kachanov, 1992 ; Mauge and Kachanov, 1992 ; Fares, 1993 ; Hu and Huang, 1993 ; NematNasser and Yu, 1993; Huang et al., 1994 ; Ju and Chen, 1994 ; Mauge and Kachanov, 1994 ; Sayers and Kachanov, 1995).

A crucial problem is the calculation of the macroscopic stiffness tensor of elastic materials intersected by various types of random or periodic crack systems. This problem has been systematically explored during the last two decades and effective methods of calculation of the overall elastic moduli of such materials have been developed, based on application of various methods for composite materials, such as the self-consistent scheme and the differential scheme. Crack arrays of various configurations have been analyzed. Variational bounds on the effective elastic moduli of crack materials have also been obtained (Kachanov, 1992, 1993). Highly accurate numerical solutions for elastic bodies with various examples of specific crack configurations have been presented (Kachanov, 1992).

There is nevertheless one serious limitation of the current knowledge. All the studies so far have dealt with stationary cracks, that is, cracks that neither propagate nor 


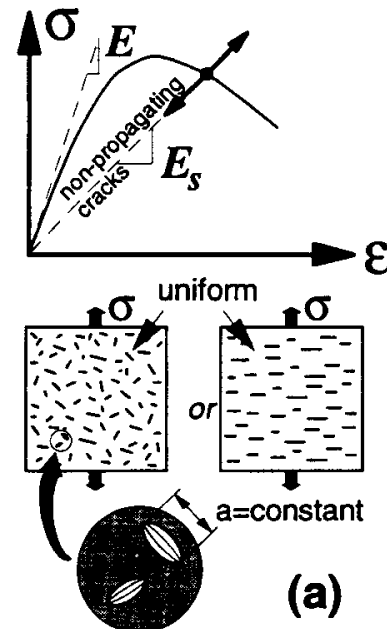

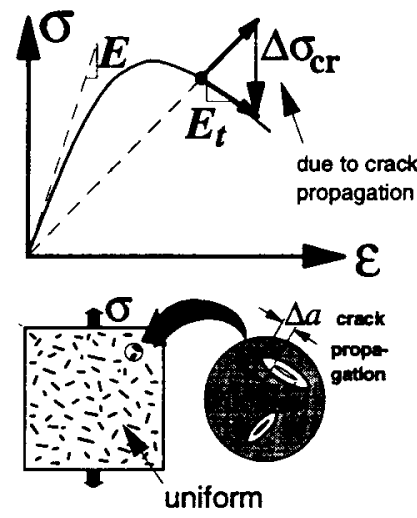

(b)

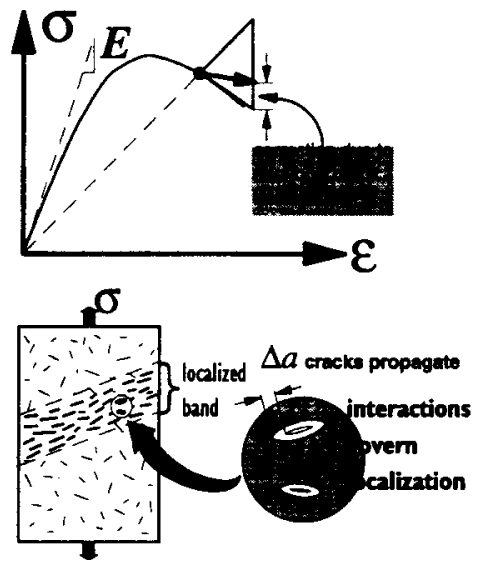

(c)

Fig. 1. (a) Effective secant moduli ; (b) tangential moduli and stress increment due to crack growth; (c) tangential stiffness of material with localizing cracks.

shorten [Fig. 1(a)]. This means that, in the context of response of a material with growing damage illustrated by the curve in Fig. 1(a), the existing formulations predict only the secant elastic moduli [such as $E_{\mathrm{s}}$ in Fig. 1(a)]. Such information does not suffice for calculating the response of a body with progressing damage due to cracking. The objective of the present paper is to analyze the response of microcracked materials taking into account that in reality the cracks can grow or shorten during loading.

By calculating the response of a material with cracks that can grow or shorten, it is possible to determine the tangential moduli, exemplified by $E_{\mathrm{t}}$ in Fig. 1(b). Knowledge of such moduli makes it possible, for a given strain increment, to determine the inelastic stress drop $\Delta \sigma_{\mathrm{cr}}$ [Fig. 1(b)]. This is obviously a harder problem than the calculation of the secant moduli, because one must impose the additional conditions that the growing cracks must remain critical, i.e. their energy release rates must remain equal to the fracture energy $G_{\mathrm{f}}$ of the material, and that, for shortening cracks in which the crack faces are coming into contact, the energy release rate must be 0 .

The problem of tangential moduli of a material with nonstationary cracks will be addressed in the paper, expanding a preliminary conference presentation of Bažant and Prat (1995). The problem will be addressed here under the assumption that, despite the growth of the cracks, their statistical distribution in space remains uniform. In other words, it will be assumed that the cracks do not localize into bands or some other domains. In that case, the effective secant as well as tangent elastic moduli calculated for a uniformly deforming specimen can be regarded as local material properties.

Knowledge of the secant and tangential moduli is of course still insufficient to predict the response of a structure with growing cracks. It is now well known that softening damage caused by cracking tends to localize into cracking bands or other regions. The localization of cracking is caused and governed mainly by interactions among propagating cracks. The interactions cause the average behavior of a rep- 


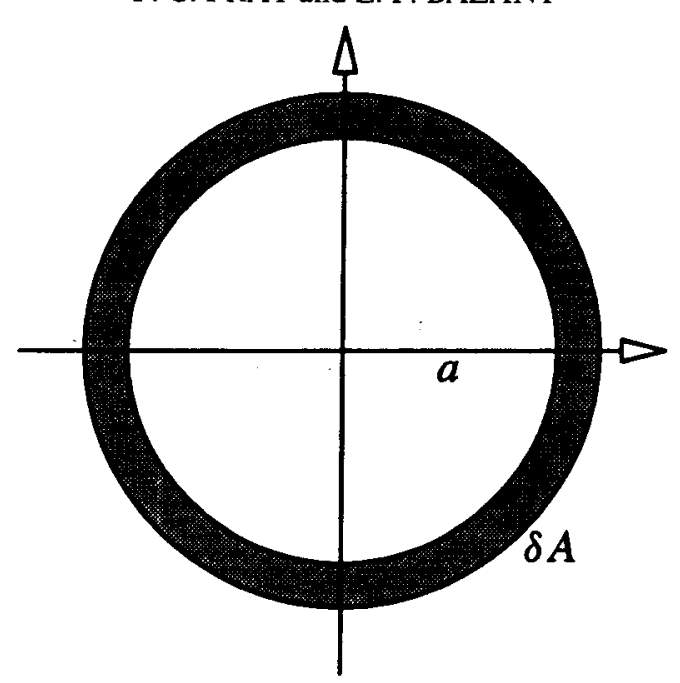

Fig. 2. Geometry of a circular crack.

resentative volume of the material with cracks not to follow the local stress-strain curve for growing cracks but to follow a slope that is either smaller or larger, as shown in Fig. 1(c). This problem has recently been analyzed and an integral equation in space governing the nonlocal behavior of such material has been formulated (Bažant, 1994; Bažant and Jirásek, 1994; Jirásek and Bažant, 1994) on the basis of continuum smoothing of a matrix equation for crack interactions. A complete solution of this problem, which obviously requires as input the information about the secant and tangential moduli, is beyond the scope of the present paper but will need to receive attention in the future.

\section{EQUIVALENT ELASTIC MODULUS OF A MATERIAL WITH UNIFORMLY DISTRIBUTED CRACKS}

We consider a representative volume $V$ of an elastic material containing on the microscale many cracks (microcracks). On the macroscale, we imagine the cracks to be smeared and the material to be represented by an approximately equivalent homogeneous continuum whose local deformation within the representative volume can be considered approximately homogeneous over the distance of several crack sizes. Let $\varepsilon$ and $\sigma$ be the average strain tensor and average stress tensor within this representative volume. On the microscale, the cracks cause the fields of microstrains and microstresses (i.e. the actual strains and stresses) to be very complex and exhibit high fluctuations about the macrostress and macrostrain. But we do not attempt to describe these fluctuations.

To obtain a simple formulation, we consider a system of circular cracks of the same radius, $a$. The cracks are uniformly distributed in space, with every crack orientation equally frequent (Fig. 2). Lest it be thought that the assumption of circular cracks 
might be overly restrictive, note that under certain conditions stated by Kachanov (Kachanov, 1993), circular cracks can be used as an approximation of elliptical cracks for the purpose of calculating the secant moduli. For that purpose, and for the case of the self-consistent scheme or the differential scheme, circular cracks of nonuniform radius can be approximated by uniform cracks for which the cube of the radius is the average of the cubes of the radii of the individual cracks. However, it is not known whether such approximations are acceptable if the cracks grow during the loading. If there are large differences in the crack size, the approximation by a uniform crack radius is probably invalid.

Fracture analysis must be based on energy. To this end we will need the complementary energy per unit volume of the material, $\Pi^{*}$, which may be written as

$$
\Pi^{*}(\boldsymbol{\sigma}, \bar{a})=\frac{1}{2} \boldsymbol{\sigma}: \mathbf{C}(\bar{a}): \boldsymbol{\sigma}
$$

in which $\mathbf{C}(\bar{a})$ is the macroscopic secant compliance tensor of the material with the cracks.

To derive the macroscopic tangential compliance tensor of the material, the cracks must be allowed to grow during the prescribed strain increment $\Delta \varepsilon$. This means that the energy release rate per unit length of the front edge of one crack, $\mathscr{G}_{1}$, must be equal to its critical value, i.e. to the fracture energy $G_{\mathrm{f}}$ of the material. However, it would be too complicated, and for the approximation of macroscopic behavior unnecessary, to consider that each growing crack satisfies the condition $\mathscr{G}_{1}=G_{\mathrm{f}}$ exactly.

For the sake of simplicity, we will enforce the condition of criticality of cracks only in a global (weak) sense. We require that the total energy release rate of all the cracks within the representative volume be equal to their combined energy dissipation rate, although we allow the energy dissipation rate per unit length of the front edge of an individual crack to differ from $G_{\mathrm{f}}$. This condition is a global statement of the law of conservation of energy (the first law of thermodynamics).

Let $n$ be the number of cracks per unit volume of the material with cracks, characterizing the representative volume. The surface area of one circular crack of radius $a$ is $A=\pi a^{2}$ and its change when the crack radius increases by $\delta a$ is $\delta A=2 \pi a \delta a$. If the crack sizes are different, one can, as an approximation, replace the actual crack radii $a_{i}$ by the effective crack radius $\bar{a}=\left(\sum_{i=1}^{n} a_{i}^{3} / n\right)^{1 / 3}$ which is uniform for all the cracks. This approximation is known to be acceptable also for stationary cracks of not too different sizes, and we assume it to be acceptable also for growing or shortening cracks.

The critical state of crack growth is obtained when the energy release rate of all the cracks per unit volume of the material, $\mathscr{G}_{1}$, is equal to the rate of total energy dissipation by all the cracks in the unit volume, i.e.

$$
\frac{\partial \Pi^{*}}{\partial \bar{a}}=2 \pi \bar{a} n G_{\mathrm{f}}
$$

Substituting $\Pi^{*}=\Pi^{*}(\sigma, \bar{a})$ from $(1)$, one obtains

$$
2 \pi \bar{a} n G_{\mathrm{f}}=\frac{\partial \Pi^{*}}{\partial \bar{a}}=\sigma: \mathbf{C}: \frac{\partial \boldsymbol{\sigma}}{\partial \bar{a}}+\frac{1}{2} \boldsymbol{\sigma}: \frac{\partial \mathbf{C}}{\partial \bar{a}}: \boldsymbol{\sigma} .
$$


For the purpose of numerical analysis, this may be written in the following incremental form

$$
2 \pi \bar{a} n G_{\mathrm{f}} \Delta \bar{a}=\sigma: \mathrm{C}: \Delta \sigma+\frac{1}{2} \sigma: \frac{\partial \mathrm{C}}{\partial \bar{a}}: \sigma \Delta \bar{a} .
$$

Calling $G^{*}=2 \pi n G_{f}$, the following equation for $\Delta \bar{a}$ is obtained

$$
\boldsymbol{\sigma}: \mathbf{C}: \Delta \boldsymbol{\sigma}+\left[\frac{1}{2} \boldsymbol{\sigma}: \frac{\partial \mathbf{C}}{\partial \bar{a}}: \boldsymbol{\sigma}-G^{*} \bar{a}\right] \Delta \bar{a}=0
$$

in which $\Delta \bar{a}=\bar{a}-\bar{a}_{0}=$ crack radius increment. Thus, we obtain the following equation for $\bar{a}$

$$
\boldsymbol{\sigma}: \mathbf{C}(\bar{a}): \Delta \boldsymbol{\sigma}+\left[\frac{1}{2} \boldsymbol{\sigma}: \frac{\partial \mathbf{C}(\bar{a})}{\partial \bar{a}}: \boldsymbol{\sigma}-G^{*} \bar{a}\right]\left(\bar{a}-\bar{a}_{0}\right)=0
$$

Equation (6) can be easily solved numerically, and the current crack size $a$ is obtained. The strains are then computed from

$$
\varepsilon=\mathbf{C}(\bar{a}): \sigma .
$$

Equation (7) is explicit. No iterations within the constitutive subroutines for structural analysis by incremental loading are required.

\section{Equivalent elastic stiffness predicted by the self-consistent scheme}

The secant elastic compliance tensor $\mathbf{C}(\bar{a})$ that appears in the foregoing equations must be evaluated at each load increment as a function of the current crack radius $\bar{a}$. To this effect, various methods for evaluating the elastic constants of composites can be used. The self-consistent scheme (Budiansky and O'Connell, 1976) will be used first.

The self-consistent scheme to evaluate the effective elastic properties of a continuum with many cracks consists in calculating the loss of energy that a single crack causes in a homogeneous continuum that has the effective elastic properties (as yet unknown) of the material with many cracks (Budiansky and O'Connell, 1976). As an approximation, the effective elastic properties are a function of the crack density parameter $\rho$, which, for circular cracks, is defined as

$$
\rho=n \bar{a}^{3} .
$$

Budiansky and O'Connell (1976) obtained the relation between the crack density parameter $\rho$ and the effective elastic constants of an elastic material with many randomly located and randomly oriented cracks. These effective elastic constants, consisting of effective Young's modulus $E_{\text {eff }}$ and effective Poisson's ratio $v_{\text {eff }}$, characterize an uncracked isotropic material having approximately the same behavior as the actual cracked material. They depend on the crack density parameter $\rho$ and, therefore, on the crack size as well : $E_{\mathrm{eff}}=E_{\mathrm{eff}}(\bar{a}), v_{\mathrm{eff}}=v_{\mathrm{eff}}(\bar{a})$. The effective Poisson's ratio $v_{\mathrm{eff}}(\bar{a})$ is obtained from the equation 


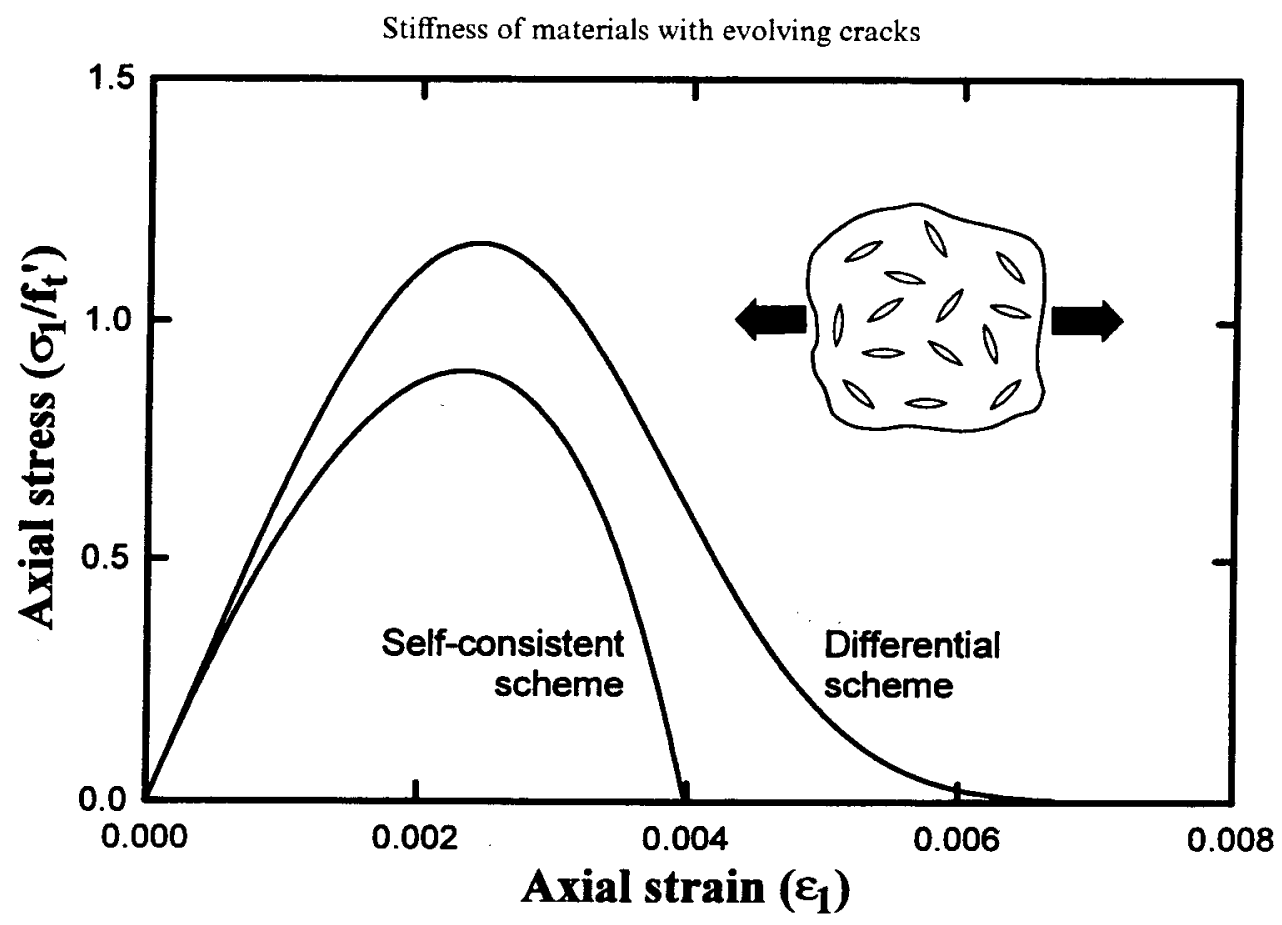

Fig. 3. Uniaxial stress-strain curve for a macroscopically isotropic randomly cracked body under uniaxial tension, using the self-consistent and differential schemes.

$$
\rho=R \frac{v-v_{\mathrm{eff}}}{1-v_{\mathrm{eff}}^{2}}
$$

where $R=45 /[16(1+3 v)]$. Solving this equation, one obtains the effective Poisson's ratio

$$
v_{\mathrm{eff}}(\bar{a})=\frac{1}{2 \rho}\left[R \pm \sqrt{R^{2}-4(R v-\rho)}\right]
$$

The effective Young's modulus is then evaluated as

$$
\frac{E_{\mathrm{eff}}(\bar{a})}{E}=1-\frac{16}{15}\left(1-v_{\mathrm{eff}}^{2}\right) \rho
$$

Figure 3 shows the results of numerical analysis performed according to the selfconsistent scheme for the case of uniaxial tension of a macroscopically uniformly strained body with random cracks. It is seen that for quasi-brittle materials such as concrete, rocks, ceramic composites, etc., which exhibit strain-softening, the selfconsistent approach yields realistic results only up to the early post-peak strains. For strain-softening at larger strains, the self-consistent scheme is inadequate. The reduction of the secant stiffness for increasing values of the crack density parameter, 


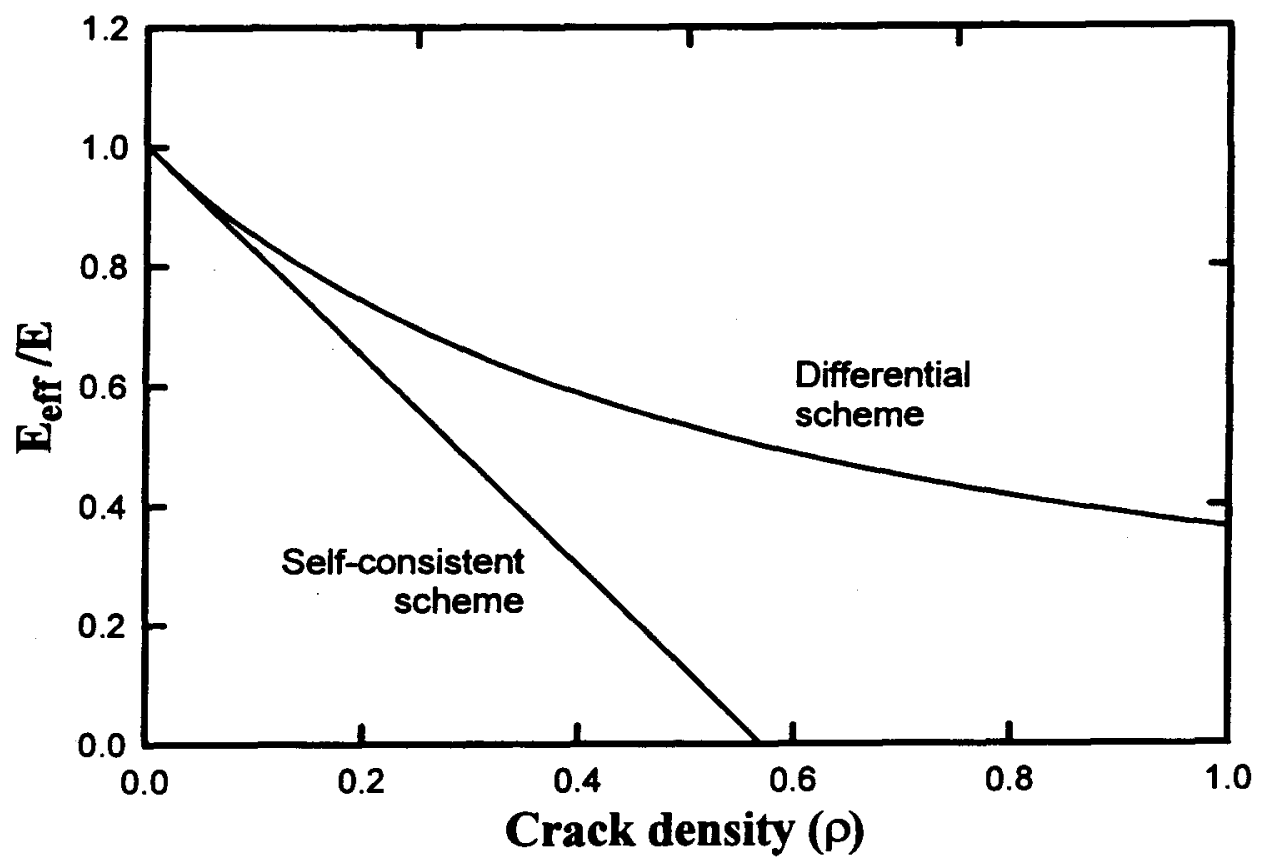

Fig. 4. Prediction of residual stiffness by self-consistent scheme (SCS) and by differential scheme (DS).

as predicted by the self-consistent scheme, is too severe. In particular, Fig. 4 shows that the self-consistent scheme predicts zero secant stiffness for a finite value of the crack density parameter, $\rho=9 / 16$. This is contradicted by tests of concrete and other quasi-brittle materials which show that the post-peak softening diagram has a very long tail (Budiansky and O'Connell, 1976; Sayers and Kachanov, 1991 ; Kachanov, 1992 ; Kachanov, 1993).

\section{Equivalent elastic stiffness predicted by the differential scheme}

To remedy the aforementioned problem, the differential scheme (Roscoe, 1952; Salganik, 1973; Zimmerman, 1985; Hashin, 1988) has been tried. In this scheme, one calculates the changes in the effective elastic moduli caused by adding to the existing cracks just one crack. Tracing such changes as the cracks are added sequentially, one by one, the prescribed number of cracks is eventually reached. More precisely, in the differential scheme, the effective elastic properties are calculated sequentially in many small steps from the energy loss caused by introducing just one crack into the equivalent homogeneous elastic material that has the elastic properties of a material with only the previously introduced cracks. In the self-consistent scheme, by contrast, the effective elastic properties are calculated in one step from the energy loss caused by introducing a single crack into an equivalent homogeneous elastic material already containing all the cracks.

For the case of circular cracks, the following relation is found [see, e.g. Hashin (1988)] 


$$
\rho=\frac{5}{8} \ln \frac{v}{v_{\mathrm{eff}}}+\frac{15}{64} \ln \frac{1-v_{\mathrm{eff}}}{1-v}+\frac{45}{128} \ln \frac{1+v_{\mathrm{eff}}}{1+v}+\frac{5}{128} \ln \frac{3-v_{\mathrm{eff}}}{3-v} .
$$

The effective Poisson's ratio, $v_{\text {eff }}(\bar{a})$, is obtained from (12) in terms of the initial Poisson's ratio $v$ of the uncracked material and the current crack density. The effective elastic modulus then is

$$
\frac{E_{\mathrm{eff}}(\bar{a})}{E}=\left(\frac{v_{\mathrm{eff}}}{v}\right)^{10 / 9}\left(\frac{3-v}{3-v_{\mathrm{eff}}}\right)^{1 / 9} .
$$

The numerical predictions of the differential scheme agree with the test results on quasi-brittle materials better than the predictions of the self-consistent scheme. Figure 3 compares the predictions for the uniaxial tensile stress-strain curve obtained with the differential and self-consistent schemes. Figure 4 gives the effective secant stiffness as a function of the crack density. The important point to note is that the differential scheme, in contrast to the self-consistent scheme, does not predict a zero secant stiffness for a finite value of the crack density parameter.

\section{EQUIVALENT ELASTIC MODULUS OF A MATERIAL WITH ARBITRARILY ORIENTED CRACKS}

So far we have considered all orientations of cracks to be equally probable, which must obviously lead to isotropic effective elastic properties. In reality, when the cracks are produced by a nonisotropic strain tensor, a preferential crack orientation must exist. So we will now assume that the crack orientations are not uniformly distributed but that there exist several families of cracks, each with a different orientation, different crack size and different crack density.

The constitutive relation of an elastic material with many random cracks that are distributed statistically uniformly in space may be written as

$$
\varepsilon=\mathrm{C}: \boldsymbol{\sigma}
$$

in which $\varepsilon, \sigma=$ macroscopic (average) strain and stress tensors, $\mathbf{C}=$ fourth-rank compliance tensor of the material with cracks, depending on $\varepsilon$, and the colon denotes a doubly contracted tensor product.

Consider now that the elastic body is intersected by $N$ families of random cracks, labeled by subscripts $\mu=1,2, \ldots, N$. Each crack family may be characterized by its spatial orientation $v_{\mu}$, its crack radius $\bar{a}_{\mu}$, and the number $n_{\mu}$ of cracks in family $\mu$ per unit volume of the material. All the cracks may approximately be considered as circular, with radius $\vec{a}_{\mu}$ in family $\mu$. Thus, the compliance tensor may be considered as the function

$$
\mathbf{C}=\mathbf{C}\left(\bar{a}_{1}, \bar{a}_{2}, \ldots, \bar{a}_{N} ; n_{1}, n_{2}, \ldots, n_{N}\right) \text {. }
$$

Approximate estimation of this function has been extensively reviewed by Kachanov and co-workers (Kachanov, 1992; Kachanov, 1993; Sayers and Kachanov, 1991 ; Kachanov et al., 1994). 
The incremental constitutive relation can be obtained by differentiation of (14), which yields

$$
\Delta \boldsymbol{\varepsilon}=\mathbf{C}: \Delta \boldsymbol{\sigma}+\sum_{\mu=1}^{N} \frac{\partial \mathbf{C}}{\partial \bar{a}_{\mu}}: \sigma \Delta \bar{a}_{\mu}+\sum_{\mu=1}^{N} \frac{\partial \mathbf{C}}{\partial n_{\mu}}: \sigma \Delta n_{\mu},
$$

where $\Delta$ denotes small increments over a loading step.

Our analysis will be restricted to the case when the number of cracks in each family is not allowed to change $\left(\Delta n_{\mu}=0\right.$, i.e. no new cracks are created and no existing cracks are allowed to vanish). However, for a material such as concrete, the exclusion or creation of new cracks is not as restrictive an assumption as it might seem. This material is full of microscopic cracks since its creation. Taken literally, new cracks in concrete are in fact never created. Every crack starts by the growth of an existing microscopic crack, which has been in the material since the beginning. For other materials, the new cracks that are being nucleated can be assumed, for all practical purposes, to have existed since the material creation with a sufficiently tiny size such that the crack of that tiny size has only a negligible effect on the compliance of the material.

With the number of cracks constant, the third term on the right-hand side of (16) vanishes, yielding

$$
\Delta \boldsymbol{\varepsilon}=\mathbf{C}: \Delta \boldsymbol{\sigma}+\sum_{\mu=1}^{N} \frac{\partial \mathbf{C}}{\partial \bar{a}_{\mu}}: \boldsymbol{\sigma} \Delta \bar{a}_{\mu}
$$

The crack radius increments $\Delta \bar{a}_{\mu}$ must be determined in conformity to the laws of fracture mechanics. Let us assume that the cracks (actually microcracks) follow linear elastic fracture mechanics (LEFM). This means that the crack front is assumed to be sharp, with no sizable cohesive zone nor fracture process zone, and the fracture energy $G_{\mathrm{f}}$ characterizing the energy dissipation rate is assumed to be a constant. This seems not a very restrictive assumption, because the main cause of the deviations of the macroscopic fracture behavior of a quasi-brittle material from LEFM is not the deviation of the behavior of the individual microcracks from LEFM. Rather, it is the existence of a large zone of microcracks (fracture process zone) at the front of a major macrocrack, along with the variations of the size of this zone and of the number and size of the microcracks in this zone.

According to the principle of conservation of energy (or the condition of a critical state of crack propagation), the energy release rate of the cracks must be equal to the fracture energy of the material, $G_{\mathrm{f}}$. Strictly speaking, each of the random microcracks in the material should follow this energy balance condition, which means that the energy release rate of each crack per unit length of its front edge should be equal to $G_{\mathrm{f}}$. However, to make the problem tractable, we impose (as before) the energy balance condition only in the global (weak) sense. We require the combined energy release rate of all the cracks in each crack family to match the combined energy dissipation rate of all the cracks in that family, as calculated from the fracture energy $G_{\mathrm{f}}$ of the material, but we allow the energy release rate of an individual crack to differ from its energy dissipation rate. This means that we have the following $N_{\mathrm{g}}$ conditions 


$$
\frac{\partial \Pi^{*}}{\partial \bar{a}_{\mu}}=2 \pi \bar{a}_{\mu} n_{\mu} G_{\mathrm{f}} \quad \text { for } \Delta \bar{a}_{\mu}>0 \quad\left(\mu=1,2, \ldots, N_{\mathrm{g}}\right),
$$

in which $N_{\mathrm{g}}$ is the number of families of growing cracks, and $\Pi^{*}$ is the complementary energy of the cracked material per unit volume of the material. (Repetition of subscript $\mu$ in this and subsequent equations does not imply summation.)

When the cracks are shortening, their faces are coming in contact, which requires no energy. Therefore, for shortening cracks

$$
\frac{\partial \Pi^{*}}{\partial \bar{a}_{\mu}}=0 \quad \text { for } \Delta \bar{a}_{\mu}<0 \quad\left(\mu=N_{\mathrm{g}}+1, \ldots, N_{\mathrm{s}}\right),
$$

where $N_{\mathrm{s}}$ is the number of all the families of growing and shortening cracks. For the remaining crack families $\left(\mu=N_{\mathrm{s}}+1, \ldots, N\right)$ no equations are necessary since for them $\Delta \bar{a}_{\mu} \equiv 0$.

The complementary energy per unit volume of the microcracked material is expressed as

$$
\Pi^{*}=\frac{1}{2} \boldsymbol{\sigma}: \mathbf{C}: \boldsymbol{\sigma}
$$

Differentiating (20), the following system of equations is obtained

$$
\frac{\partial \Pi^{*}}{\partial \bar{a}_{\mu}}=\boldsymbol{\sigma}: \mathbf{C}: \frac{\partial \boldsymbol{\sigma}}{\partial \bar{a}_{\mu}}+\frac{1}{2} \boldsymbol{\sigma}: \frac{\partial \mathbf{C}}{\partial \bar{a}_{\mu}}: \boldsymbol{\sigma} \quad(\mu=1,2, \ldots, N) .
$$

Substituting here (18) of (19), and considering also that cracks $\mu=N_{\mathrm{s}}+1, \ldots, N$ neither shorten nor grow (i.e. are stationary), we finally obtain the following incremental relations which must be satisfied by the crack radius increments (positive or negative, or 0 )

$$
\begin{gathered}
\boldsymbol{\sigma}: \mathbf{C}: \Delta \boldsymbol{\sigma}+\left(\frac{1}{2} \boldsymbol{\sigma}: \frac{\partial \mathbf{C}}{\partial \bar{a}_{\mu}}: \boldsymbol{\sigma}-2 \pi \bar{a}_{\mu} n_{\mu} G_{\mathrm{f}}\right) \Delta \bar{a}_{\mu}=0 \quad\left(\Delta \bar{a}_{\mu}>0, \mu=1, \ldots, N_{\mathrm{g}}\right), \\
\boldsymbol{\sigma}: \mathbf{C}: \Delta \boldsymbol{\sigma}+\frac{1}{2} \boldsymbol{\sigma}: \frac{\partial \mathbf{C}}{\partial \bar{a}_{\mu}}: \boldsymbol{\sigma} \Delta \bar{a}_{\mu}=0 \quad\left(\Delta \bar{a}_{\mu}<0, \mu=N_{\mathrm{g}}+1, \ldots, N_{\mathrm{s}}\right) \\
\Delta \bar{a}_{\mu}=0 \quad\left(\mu=N_{\mathrm{s}}+1, \ldots, N\right) .
\end{gathered}
$$

The first equation applies to the families of growing cracks, the second to the families of shortening cracks, and the third to the families of stationary cracks.

The constitutive law of (17) and the energy equilibrium conditions of (22) together represent a system of $N+6$ equations for the increments of crack radii in $N$ crack families of different orientations and 6 increments of stress tensor components, $\Delta \sigma$. If the strain tensor increment $\Delta \varepsilon$ is prescribed, these $N+6$ unknowns can be solved from this system of equations.

The tangential stiffness tensor can be obtained by solving the stress increments for all the cases in which a unit value is assigned to each component of $\Delta \sigma$, with all the other components being 0 . 
To solve the problem, we need the column matrix (vector) $\mathbf{a}=\left\{\bar{a}_{\mu}\right\}$ of the current crack radii (sizes) for the crack families of all orientations $\mu$ as a function of the prescribed strain. We also need to calculate the effective compliance, which in general depends on the current a (15), and the stress tensor, which depends on the strain tensor $\varepsilon$ and on the current $\mathbf{a}$.

To do that, we must have the means to evaluate the effective secant stiffness $\mathbf{C}$ as a function of the column matrix of the crack radii. For its simplicity, we will use a technique developed by Sayers and Kachanov (1991). To this end, they introduced a symmetric second-order crack density tensor (Vakulenko and Kachanov, 1971; Kachanov, 1980, 1987b) defined by

$$
\boldsymbol{\alpha}=\sum_{\mu=1}^{N} n_{\mu} \bar{a}_{\mu}^{3} v_{\mu} \otimes v_{\mu}
$$

This tensor represents a tensorial generalization of the scalar crack density, accounting for the crack orientation. In particular, for the case of random cracks, it can be shown that

$$
\operatorname{tr} \alpha=\alpha_{k k}=\sum_{\mu=1}^{N} n_{\mu} \bar{a}_{\mu}^{3}=\rho
$$

where $\rho$ is the crack density parameter of an isotropic randomly cracked medium, as defined in (8).

The effective secant compliance $\mathbf{C}$ can be derived from an elastic potential $F$ which may be considered as a function of the crack density tensor $\alpha$ in addition of the stress tensor $\boldsymbol{\sigma}$ :

$$
\varepsilon=\frac{\partial \mathbf{F}(\boldsymbol{\sigma}, \boldsymbol{\alpha})}{\partial \boldsymbol{\sigma}}=\mathbf{C}: \boldsymbol{\sigma}
$$

Also,

$$
\mathbf{C}=\mathbf{C}^{\circ}+\mathbf{C}^{\mathrm{cr}}
$$

in which $\mathbf{C}^{\circ}$ is the elastic compliance of uncracked elastic material and $\mathbf{C}^{\mathrm{cr}}$ is the additional compliance due to the crack system.

The elastic potential $F(\sigma, \alpha)$ can be expanded into a tensorial power series. According to the Cayley-Hamilton theorem, this expansion can always be reduced to a cubic tensor polynomial. Sayers and Kachanov (1991) proposed to approximate potential $\mathbf{F}$ by a tensor polynomial that is quadratic in $\sigma$ and linear in $\alpha$

$$
F(\boldsymbol{\sigma}, \boldsymbol{\alpha})=\frac{1}{2} \boldsymbol{\sigma}: \mathbf{C}^{\circ}: \boldsymbol{\sigma}+\eta_{1}(\boldsymbol{\sigma}: \boldsymbol{\alpha}) \operatorname{tr} \boldsymbol{\sigma}+\eta_{2}(\boldsymbol{\sigma} \cdot \boldsymbol{\sigma}): \boldsymbol{\alpha} .
$$

Here $\eta_{1}$ and $\eta_{2}$ can, in general, be nonlinear functions of the invariants of the crack density tensor $\alpha$. For the sake of simplicity, we assume $\eta_{1}$ and $\eta_{2}$ to depend only on the first invariant $\rho=\operatorname{tr} \alpha=\Sigma_{\mu} n_{\mu} \bar{a}_{\mu}^{3}$ of $\alpha$ (Sayers and Kachanov, 1991). 
Equation (25) with (26) provides, in subscript notation

$$
\varepsilon_{i j}=\left[C_{i j k l}^{\mathrm{o}}+C_{i j k l}^{\mathrm{cr}}\right] \sigma_{k l}=\left[C_{i j k l}^{\mathrm{o}}+\eta_{1}\left(\alpha_{i j} \delta_{k l}+\alpha_{k l} \delta_{i j}\right)+\eta_{2}\left(\alpha_{i k} \delta_{j l}+\alpha_{j l} \delta_{i k}\right)\right] \sigma_{k l}
$$

where the subscripts refer to Cartesian coordinates $x_{i}(i=1,2,3)$. Note that this is the most general possible tensor that is linear in $\alpha_{i j}$. Equation (25) may be written as $\boldsymbol{\varepsilon}=\mathbf{C}: \boldsymbol{\sigma}$ where $\boldsymbol{\varepsilon}=\left(\varepsilon_{11}, \varepsilon_{22}, \varepsilon_{33}, 2 \varepsilon_{12}, 2 \varepsilon_{23}, 2 \varepsilon_{31}\right)^{T}$ and $\boldsymbol{\sigma}=\left(\sigma_{11}, \sigma_{22}, \sigma_{33}, \sigma_{12}, \sigma_{23}, \sigma_{31}\right)^{T}$ are the column matrices of strains and stresses and $(\cdot)^{T}$ denotes transpose of a matrix. The additional secant compliance $\mathbf{C}^{\mathrm{cr}}$ due to the cracks may then be expressed as

$\mathbf{C}^{\mathrm{cr}}=$

$$
\left[\begin{array}{cccccc}
2\left(\eta_{1}+\eta_{2}\right) \alpha_{11} & \eta_{1}\left(\alpha_{11}+\alpha_{22}\right) & \eta_{1}\left(\alpha_{11}+\alpha_{33}\right) & 2\left(\eta_{1}+\eta_{2}\right) \alpha_{12} & 2 \eta_{1} \alpha_{23} & 2\left(\eta_{1}+\eta_{2}\right) \alpha_{31} \\
& 2\left(\eta_{1}+\eta_{2}\right) \alpha_{22} & \eta_{1}\left(\alpha_{22}+\alpha_{33}\right) & 2\left(\eta_{1}+\eta_{2}\right) \alpha_{12} & 2\left(\eta_{1}+\eta_{2}\right) \alpha_{23} & 2 \eta_{1} \alpha_{31} \\
& & 2\left(\eta_{1}+\eta_{2}\right) \alpha_{33} & 2 \eta_{1} \alpha_{12} & 2\left(\eta_{1}+\eta_{2}\right) \alpha_{23} & 2\left(\eta_{1}+\eta_{2}\right) \alpha_{31} \\
& & & 2 \eta_{2}\left(\alpha_{11}+\alpha_{22}\right) & 2 \eta_{2} \alpha_{13} & 2 \eta_{2} \alpha_{23} \\
& & & & 2 \eta_{2}\left(\alpha_{11}+\alpha_{33}\right) & 2 \eta_{2} \alpha_{12} \\
& & & & & 2 \eta_{2}\left(\alpha_{22}+\alpha_{33}\right)
\end{array}\right] .
$$

The functions $\eta_{1}(\rho)$ and $\eta_{2}(\rho)$ can be obtained by taking the particular form of the preceding formulation for the case of random cracks, and equating the results to those obtained with the differential scheme, as shown in the preceding section.

For a uniform frequency distribution of crack orientations, $\alpha_{11}=\alpha_{22}=\alpha_{33}=\rho / 3$. Combining this with (28) and (26), we obtain the following system of equations for $\eta_{1}$ and $\eta_{2}$

$$
\begin{gathered}
\frac{1}{E_{\mathrm{eff}}}-\frac{1}{E}=\frac{2}{3}\left(\eta_{1}+\eta_{2}\right) \rho, \\
\frac{1}{G_{\mathrm{eff}}}-\frac{1}{G}=\frac{1}{3} \eta_{2} \rho,
\end{gathered}
$$

from which $\eta_{1}$ and $\eta_{2}$ can be solved

$$
\begin{aligned}
& \eta_{1}=-\frac{3}{2 \rho}\left(\frac{v_{\mathrm{eff}}}{E_{\mathrm{eff}}}-\frac{v}{E}\right), \\
& \eta_{2}=\frac{3}{2 \rho}\left(\frac{1+v_{\mathrm{eff}}}{E_{\mathrm{eff}}}-\frac{1+v}{E}\right) .
\end{aligned}
$$

Substituting the values of $E_{\text {eff }}$ and $v_{\text {eff }}$ from (12) and (13) for $E_{\text {eff }}$ and $v_{\text {eff }}$ in (30), we can determine $\eta_{1}$ and $\eta_{2}$. Furthermore, substituting these values into (28) and 
incrementing $\mathbf{C}$, we get the current effective secant compliance tensor, which is needed to solve $\Delta \bar{a}_{\mu}$ from the following system of equations

$$
\begin{array}{lll}
\boldsymbol{\sigma}: \mathbf{C}: \Delta \boldsymbol{\sigma}+\left[\frac{1}{2} \boldsymbol{\sigma}: \frac{\partial \mathbf{C}}{\partial \bar{a}_{\mu}}: \boldsymbol{\sigma}-G_{\mu}^{*} \bar{a}_{\mu}\right] \Delta \bar{a}_{\mu}=0 & \mu=1, \ldots, N_{\mathrm{g}} \\
\boldsymbol{\sigma}: \mathbf{C}: \Delta \boldsymbol{\sigma}+\left[\frac{1}{2} \boldsymbol{\sigma}: \frac{\partial \mathbf{C}}{\partial \bar{a}_{\mu}}: \boldsymbol{\sigma}\right] \Delta \bar{a}_{\mu} & =0 & \mu=N_{\mathrm{g}}+1, \ldots, N_{\mathrm{s}}, \\
\Delta \bar{a}_{\mu} & =0 & \mu=N_{\mathrm{s}}+1, \ldots, N, \\
\mathbf{C}: \Delta \boldsymbol{\sigma}+\sum_{\mu} \frac{\partial \mathbf{C}}{\partial \bar{a}_{\mu}}: \boldsymbol{\sigma} \Delta \bar{a}_{\mu} & =\Delta \boldsymbol{\varepsilon} &
\end{array}
$$

in which $G_{\mu}^{*}=2 \pi n_{\mu} G_{\mathrm{f}}$.

Equation (31) represents a system of $n+6$ linear equations for the stress tensor increment $\Delta \sigma$ and the increment of the column matrix of the crack radii, a, during a loading step. This system can be solved if the strain tensor increment $\Delta \varepsilon$ is given.

\section{NUMERICAL IMPLEMENTATION AND ALGORITHM}

In the present model, the growth and opening of the cracks of each family has the effect of increasing the compliance. This property is shared by a broad class of material models with a discrete set of cracks or other discontinuities of different orientations, intended for use in large-scale finite element programs with incremental loading. The experience with the numerical algorithms for such models can be exploited for the present model.

We aim at applications in large-scale finite element programs, in which the constitutive subroutine is used in each loading step to calculate the stress tensor increment from the assumed strain tensor increment. Whether, in the current loading step, each family of cracks is growing, shortening or remaining stationary cannot be determined a priori, before solving the linear equation system (31) for the crack radii increments and stress increments corresponding to the assumed strains in the current loading step. An assumption on the type of response of each family (growing, shortening or stationary) must be made first, before the equation system can be solved. After solving this equation system, the assumptions about the type of response of all crack families must be checked and updated. The calculation of the stress increments from the strain increments is then iterated. The computation must of course be aborted if the iterations do not lead to agreement with the assumed type of response of the crack families. However, numerical experience showed that the iterations converged well in all the examples cited below, and the convergence was in fact rapid.

So, if the present formulation is implemented in a finite element program, the solution is not explicit at the constitutive level because it must be predicted in advance whether the cracks of each family grow, shorten or remain stationary and the prediction must then be reconciled with the calculation results iteratively.

An explicit solution, however, is possible at the cost of lower accuracy. In that case, 
the assumption about the type of response of the cracks (growing, shortening or stationary) in the current loading step is made always on the basis of the calculation results in the preceding step. If the loading step is reduced, the error caused by delaying the determination of the crack status by one loading step still approaches zero, but the approach to zero is slower than in the aforementioned solution with iterations in each loading step.

Although the crack families can in reality have infinitely many possible orientations, for the purpose of numerical analysis it is assumed that they can have only a finite set of discrete orientations of the crack normals. These orientations may be represented by points on a unit sphere. Obviously these points should be distributed over the sphere as uniformly as possible. A perfectly uniform distribution is obtained if these points are the vertices of a regular polyhedron inscribed to the sphere. Unfortunately, a regular polyhedron cannot have more than 20 vertices (or 10 per hemisphere), which is not enough for a sufficiently accurate representation of post-peak strain softening (Bažant and Oh, 1985, 1986).

Therefore, one must accept a certain nonuniformity of the distribution of these points. The question of how to place these points on the sphere optimally and what should be the optimum weights associated with different points represents mathematically the problem of determining an optimal Gaussian quadrature formula for the surface of a sphere. This problem, which is important to several fields of physics, has been intensely studied by mathematicians and has also been tackled in the mechanics of continuum damage in connection with the so-called microplane model (Bažant and Oh, 1985, 1986).

Many different Gaussian integration formulas for the surface of a sphere, with various degrees of accuracy and numerical efficiency are available (Stroud, 1971). These formulas were reviewed by Bažant and Oh $(1985,1986)$ and a new optimal 9degree Gaussian integration formula with 21 points per hemisphere, which is more efficient than other well known formulas [see, e.g. Stroud (1971)], was developed. It provides sufficient accuracy for the microplane model even in the strain-softening range. Other Gaussian formulas with 25 and 28 points per hemisphere (Stroud, 1971) are about equally efficient. These formulas have worked well with the microplane model (Bažant and Prat, 1988a, b; Prat and Bažant, 1991 ; Bažant et al., 1996a, b), as well as with similar phenomenological models simulating multidirectional smeared continuous cracking (Carol and Prat, 1991; Carol et al., 1993; Carol and Prat, 1995).

Applying the Gaussian integration formula to the present model, one samples the infinitely many possible crack orientations with a discrete set of $N$ crack orientations determined by the spherical angles of the integration points of the formula. To ensure acceptable accuracy, $N$ must be, for a general three-dimensional stress state, at least 21 .

The algorithm for the proposed model is described by the flowchart of Fig. 5. For a more detailed description, see Carol et al. (1996).

If the material, for example concrete, is initially isotropic, the crack density (i.e. number of cracks per unit volume) will be initially uniform, i.e. $n_{\mu}=$ constant $\forall \mu \in[1, N]$. Because cracks must be allowed to grow in every direction, and because we assume no new cracks to nucleate, the initial crack density cannot be made exactly zero. A very small value must be assigned to the crack density for each orientation at the outset. 


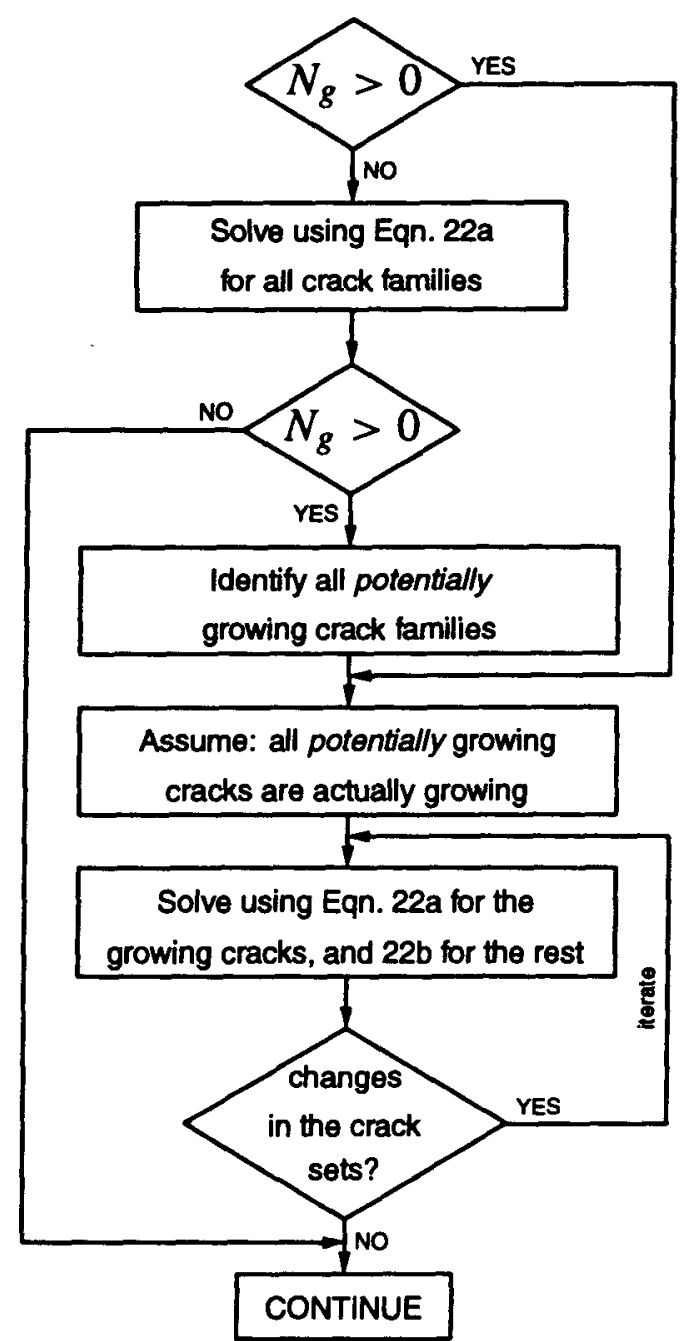

Fig. 5. Flow-chart of algorithm for the constitutive model.

If the material, for example rock or fiber composite, is initially anisotropic, the crack density will be initially different for different orientations of crack families. For precracked materials such as rocks with joints, it usually suffices to describe the directional dependence of the crack density by only a few characteristic orientations (one or two). For ease of numerical analysis, it is nevertheless convenient to assume a continuous crack density function peaking at a few dominant orientations

$$
n_{\mu}=n\left(\phi_{\mu}, \theta_{\mu}\right)=\sum_{i=1}^{N_{\mathrm{c}}} n_{i}^{*} \mathrm{e}^{-k_{i}\left[\left(\phi_{\mu}-\phi_{i}^{*}\right)^{2}+\left(\theta_{\mu}-\theta_{i}^{*}\right)^{2}\right]},
$$

where $k_{i}$ are given constants; $\phi_{\mu}$ and $\theta_{\mu}$ are spherical coordinate angles defining orientation through the unit vector normal to the cracks of family $\mu ; \phi_{i}^{*}$ and $\theta_{i}^{*}$ are the angles defining the crack orientations at which the crack density peaks, with the 


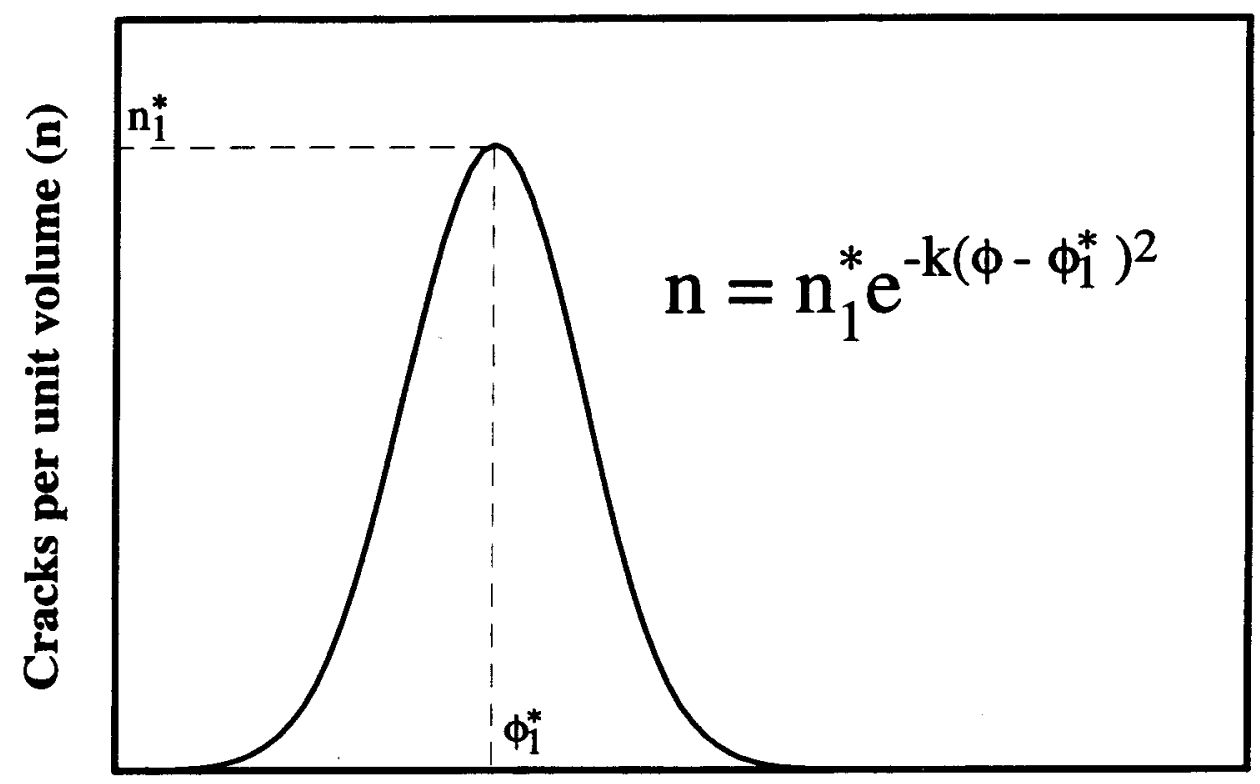

Angle of cracks relative to load direction ( $\phi)$

Fig. 6. Crack density as a function of crack orientation for one set of cracks.

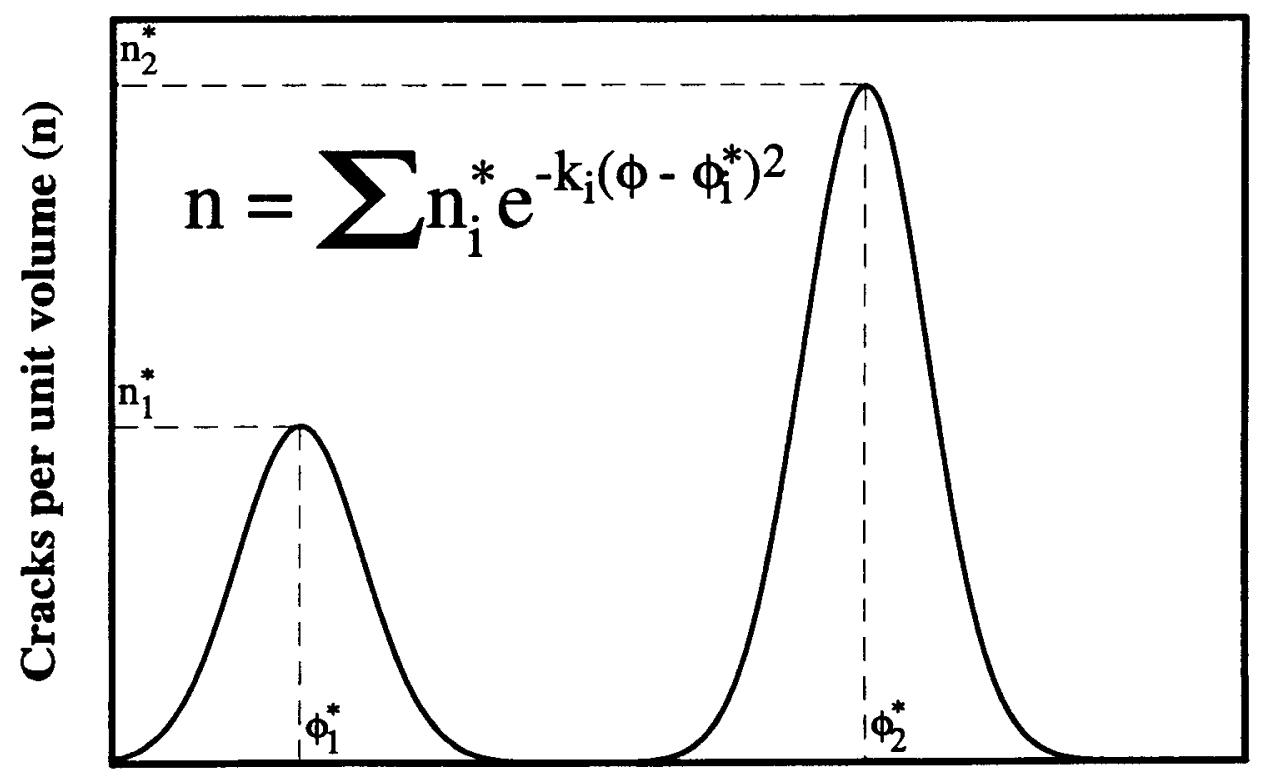

Angle of cracks relative to load direction ( $\phi)$

Fig. 7. Crack density as a function of crack orientation for two sets of cracks. 
peak value $n_{i}^{*}$; and $N_{\mathrm{c}}$ is the number of all the discrete orientations for which the peak crack densities are prescribed. Although the values of the crack density that (32) gives for the peak orientations are not strictly equal to the prescribed values $n_{i}^{*}$, the difference is negligible if the values of $k_{i}$ are specified high enough. This is being done in order to keep the crack density function in (32) reasonably simple. Examples of this function, for the case of plane stress analysis, can be seen in Fig. 6 for a single family of cracks $\left(N_{\mathrm{c}}=1\right)$ and in Fig. 7 for two families of cracks $\left(N_{\mathrm{c}}=2\right)$.

The function defined in (32) has been used to simulate the behavior of a macroscopically orthotropic material with one or two sets of crack families subjected to uniaxial compression or uniaxial tension.

\section{NUMERICAL RESULTS AND THEIR COMPARISON WITH TEST DATA FOR CONCRETE}

Figure 8 shows the results obtained when uniaxial compression is applied to an elastic material with a single family of parallel cracks. The angle of crack normals
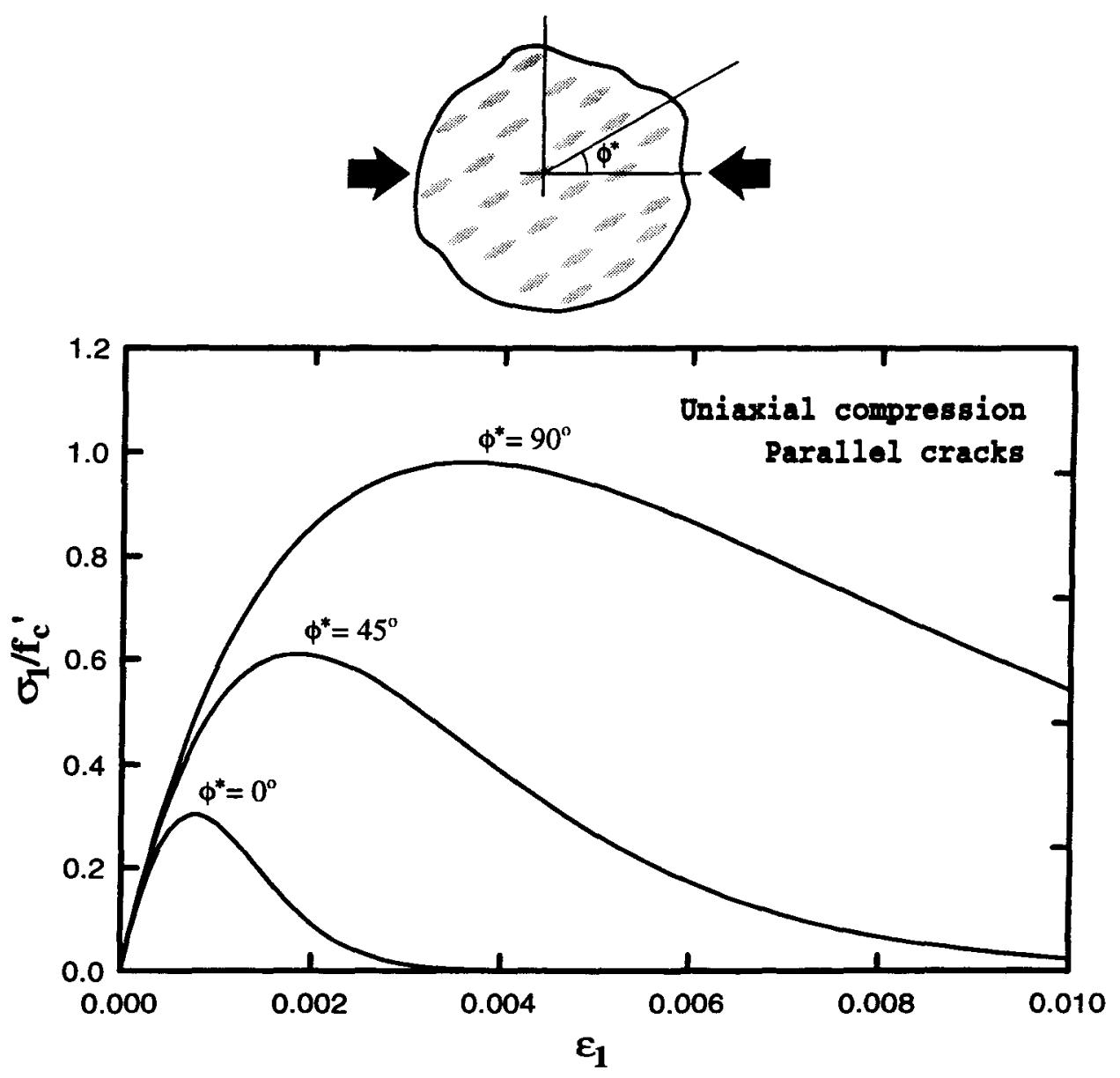

Fig. 8. Calculation results for a macroscopically orthotropic material containing one family of parallel cracks under uniaxial compression. 

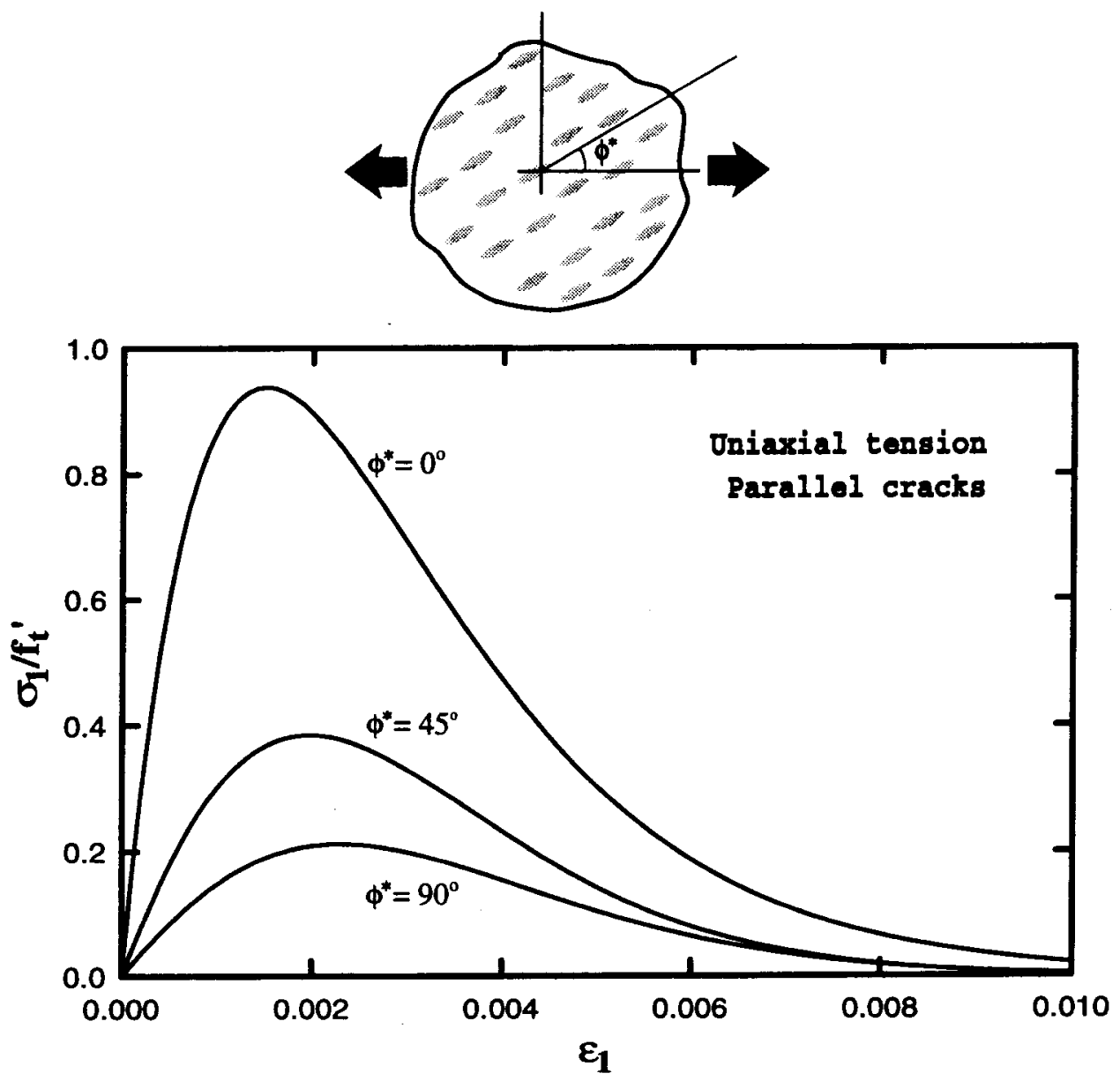

Fig. 9. Calculation results for a macroscopically orthotropic material containing one family of parallel cracks under uniaxial tension.

with the direction of compression is $\phi^{*}$. The solution has been obtained for three different values of the angle : $0^{\circ}, 45^{\circ}$ and $90^{\circ}$. As expected, when the cracks are parallel to the loading direction $\left(\phi^{*}=0^{\circ}\right)$, the calculated peak stress (strength) is the lowest and the material response is the most brittle, as indicated by the steepest post-peak decline of stress.

Figure 9 shows the results obtained when uniaxial tension is applied. In that case, the minimum strength occurs when the cracks are perpendicular to the tension direction, as known from experimental evidence.

The analysis has been repeated for an elastic material with two families of orthogonal cracks, which in general are not aligned with the tension direction $\left(\phi_{1}^{*}=30^{\circ}\right.$, $\phi_{2}^{*}=120^{\circ}$ ). The analysis has been performed for several combinations of the number of cracks $n_{1}^{*}$ and $n_{2}^{*}$ corresnonding to the two crack families. These combinations are indicated in Figs 10 and 11. Figure 10 depicts the results obtained for uniaxial compression and Fig. 11 the results for uniaxial tension. The analytical results obtained for both cases agree with the expected trends. 


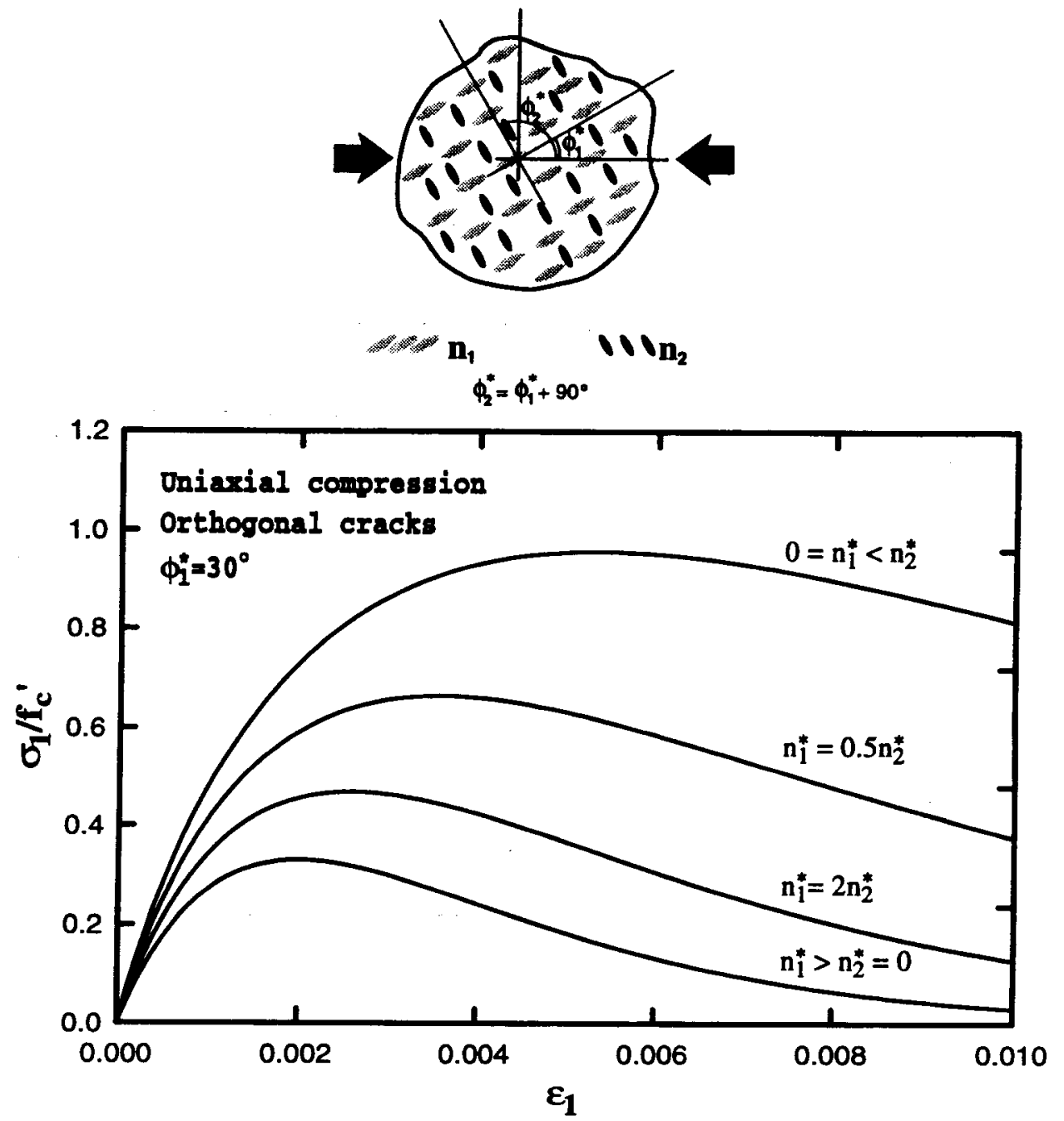

Fig. 10. Calculation results for a macroscopically orthotropic material containing two families of orthogonal cracks under uniaxial compression.

The present model has also been used to simulate the response to biaxial stress applied to an initially isotropic material. Figure 12 shows the stress-strain curves obtained for biaxial tests with different stress ratios $\sigma_{1} / \sigma_{2}$. These curves again agree with the observed behavior of brittle materials. Furthermore, combining the peaks of the curves for different stress ratios, the biaxial failure envelope (Fig. 13) is obtained. The envelope agrees well with experimental results. In particular, the ratios of the uniaxial tensile strength and of the biaxial compression strength to the uniaxial compression strength have values that are normal for concrete.

Finally, the model has been verified for the case of standard triaxial tests, in which a certain hydrostatic pressure of maximum value $\sigma_{c}$ is applied first and then uniaxial compression is superimposed. The analytical results are presented in Fig. 14, for several values of $\sigma_{\mathrm{c}}$ ranging from 0 to $10 f_{\mathrm{c}}^{\prime}$. Qualitatively, the model is able to capture the decreasing brittleness of the material for increasing hydrostatic pressure. 

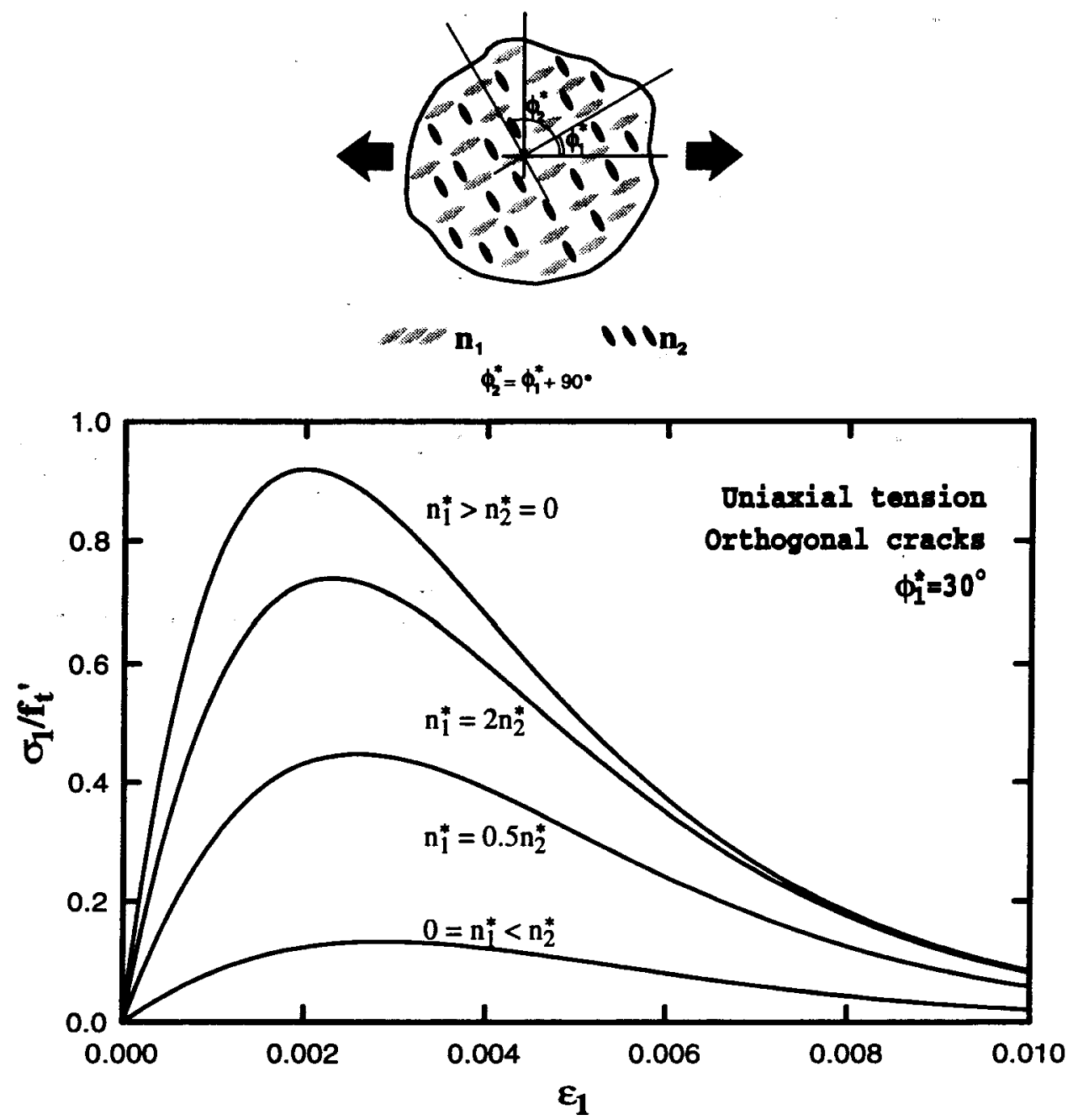

Fig. 11. Calculation results for a macroscopically orthotropic material containing two families of orthogonal cracks under uniaxial tension.

\section{CONCLUSIONS}

(1) The existing solutions in which the cracks have been considered as stationary do not suffice to model the evolution of damage in materials. It is necessary to solve the response of an elastic body to load increments in which the cracks grow while remaining critical, or shorten. This is done here for an elastic material that contains several families of cracks of either uniform or random orientation.

(2) The condition that the cracks must remain critical while they grow means that their energy release rate must be equal to their energy dissipation rate based on the fracture energy of the material. To impose this condition for each individual crack would be too complicated. To simplify the solution, this condition is imposed only in the global (weak) sense, i.e. the total energy release rate and the total energy dissipation rate of all the cracks in each crack family are required to be equal. 


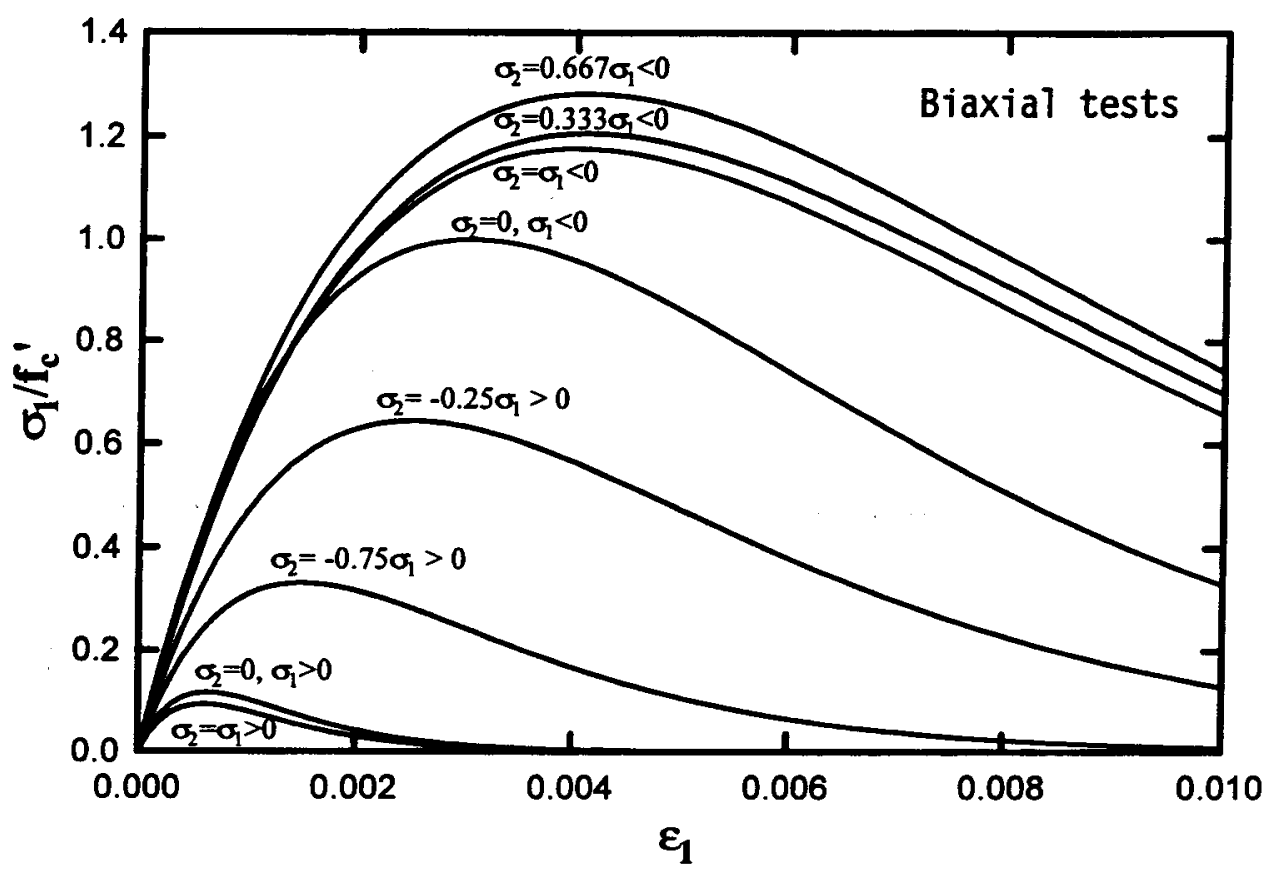

Fig. 12. Stress-strain curves from biaxial test simulations.

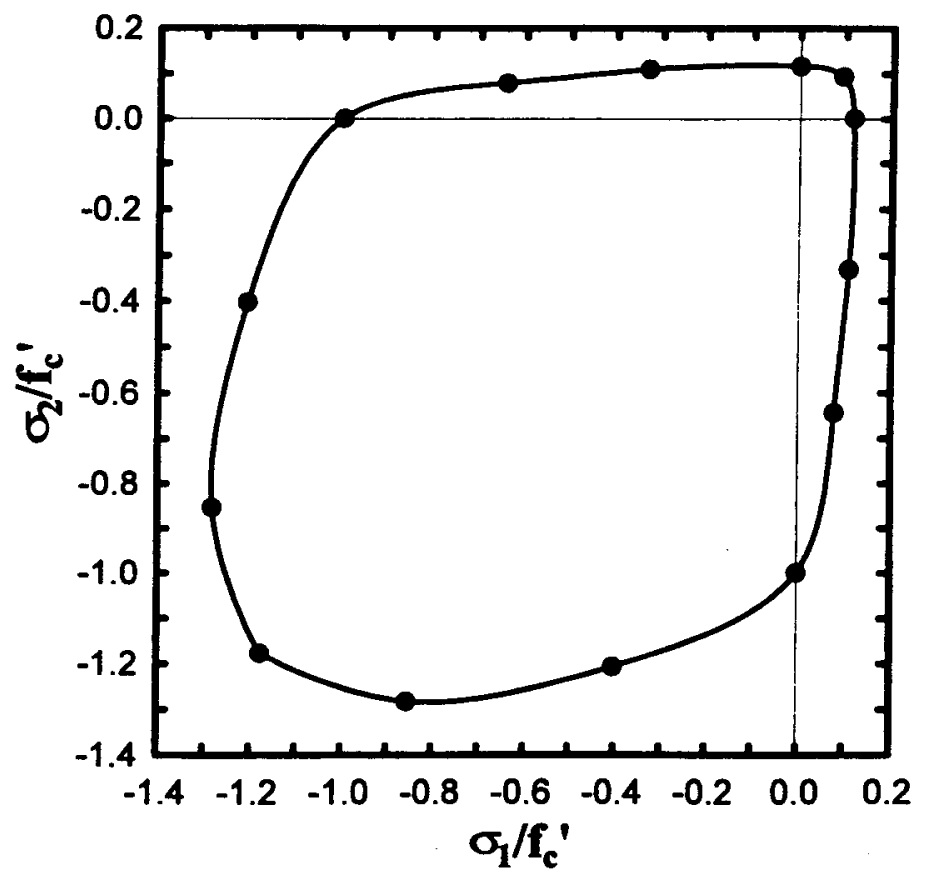

Fig. 13. Biaxial failure envelope predicted by the theory. 


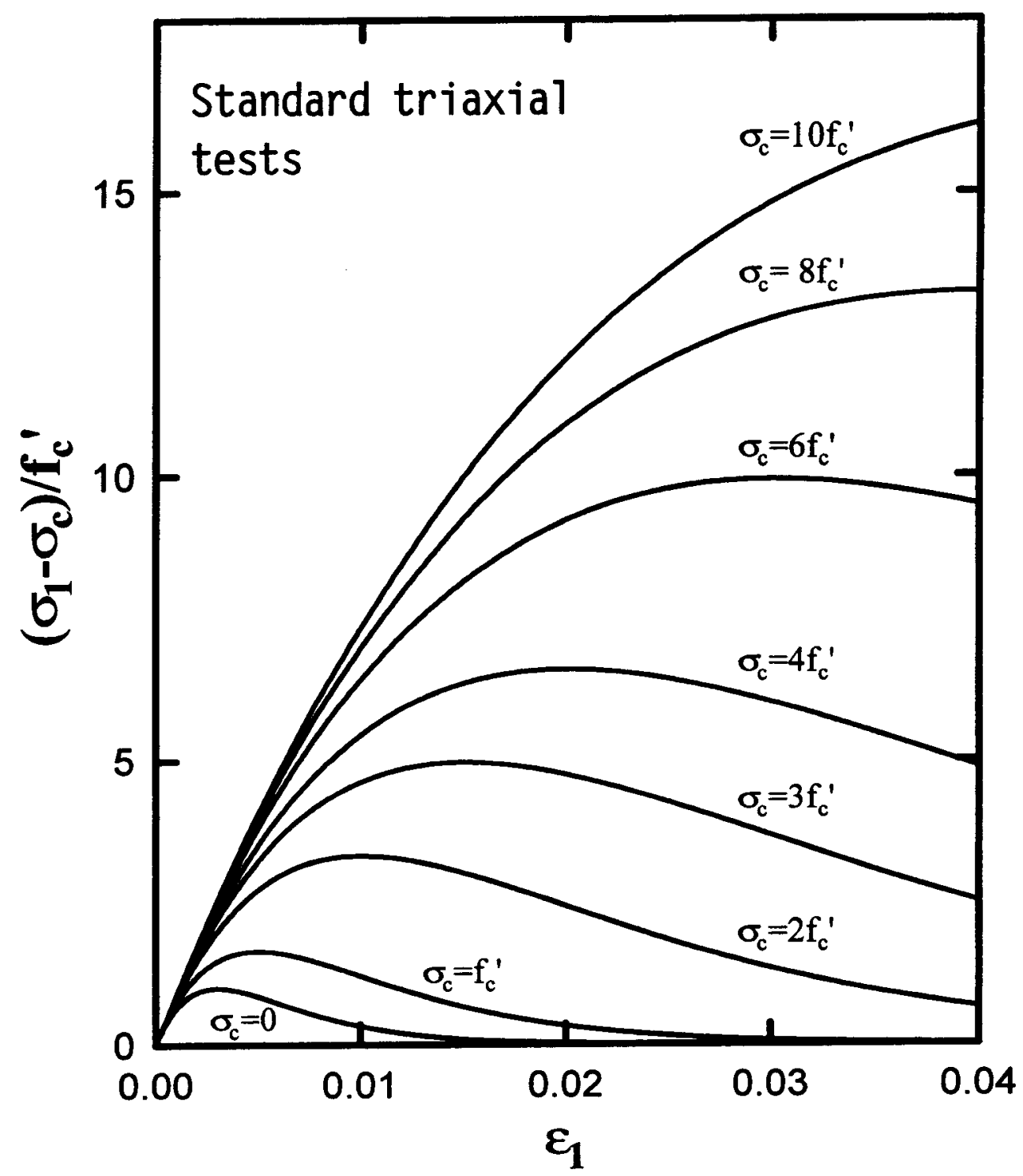

Fig. 14. Stress-strain curves from standard triaxial tests simulations.

(3) For a uniform crack distribution, it is found that the self-consistent scheme for the calculation of elastic constants of a microcracked material does not give realistic results except for deformations up to the peak load and slightly beyond. On the other hand, the differential scheme yields realistic stress-strain curves even in the post-peak strain-softening range of material response.

(4) Considering a material with several crack families of different orientations, one may use Sayers and Kachanov's approximation of the elastic potential as a polynomial that is quadratic in the macroscopic stress tensor and linear in the crack density tensor, with coefficients that are nonlinear functions of the first invariant of the crack density tensor. The values of these coefficients can be obtained with the help of the differential scheme for composite materials. For a prescribed strain tensor increment, 
this approach yields a system of $N+6$ linear equations for the increments of the stress tensor and the crack size for each of $N$ crack families.

(5) The computational results are qualitatively in good agreement with the trends observed in the testing of concrete. This indicates that the approximations made should be reasonable.

\section{ACKNOWLEDGEMENTS}

This work was carried out during a sabbatical visit of the first author to Northwestern University, financially supported by AIRE Grant 1995BEAI300213 from CIRIT (Generalitat de Catalunya), Barcelona, Spain, which is hereby gratefully acknowledged. The second author's work on the formulation of the basic theory was partly supported by ONR Grant N00001491-J-1109 to Northwestern University (monitored by Dr Yapa Rajapakse), and the application to concrete was partly supported by the ACBM Center at Northwestern University.

\section{REFERENCES}

Bažant, Z. P. (1994) Nonlocal damage theory based on micromechanics of crack interactions. ASCE J. Engng Mech. 120, 593-617 ; Addendum and Errata, 1401-1402.

Bažant, Z. P. and Jirásek, M. (1994) Damage nonlocality due to microcrack interactions: statistical determination of crack influence function. In Fracture and Damage in Quasi-Brittle Structures, ed. Z. P. Bažant, Z. Bittnar, M. Jirásek and J. Mazars, pp. 3-17. E \& FN SPON, London.

Bažant, Z. P. and Oh, B. H. (1985) Microplane model for progressive fracture of concrete and rock. ASCE J. Engng Mech. 111, 559-582.

Bažant, Z. P. and Oh, B.-H. (1986) Efficient numerical integration on the surface of a sphere. Z. angew. Math. u. Mech. (ZAMM) 66, 37-49.

Bažant, Z. P. and Prat, P. C. (1988a) Microplane model for brittle-plastic material : I. Theory. ASCE J. Engng Mech. 114, 1672-1688.

Bažant, Z. P. and Prat,.P. C. (1988b) Microplane model for brittle-plastic material: II. Verification. ASCE J. Engng Mech. 114, 1689-1702.

Bažant, Z. P. and Prat, P. C. (1995) Elastic material with growing or closing cracks : tangential stiffness. In Contemporary Research in Engineering Science, Proceedings, Eringen Medal Symposium honoring S. N. Atluri, ed. R. Batra, pp. 55-65. Society of Engineering Science, 32nd Annual Meeting, Springer Verlag.

Bažant, Z. P., Xiang, Y. and Prat, P. C. (1996a) Microplane for concrete: I. Stress-strain boundaries and finite strain. ASCE J. Engng Mech. 122, 245-262.

Bažant, Z. P., Xiang, Y., Adley, M. D., Prat, P. C. and Akers, S. A. (1996b) Microplane for concrete : II. Data delocalization and verification. ASCE J. Engng Mech. 122, 263-268.

Budiansky, B. (1965) On the elastic moduli of some heterogeneous materials. J. Mech. Phys. Solids 13, 223-227.

Budiansky, B. and O'Connell, R. J. (1976) Elastic moduli of a cracked solid. Int. J. Solids Structures 12, 81-97.

Carol, I. and Prat, P. C. (1991) Smeared analysis of concrete fracture using a microplane based multicrack model with static constraint. In Fracture Processes in Concrete, Rock and Ceramics, ed. J. G. M. van Mier, J. G. Rots and A. Bakker, pp. 619-628. E \& FN SPON, London.

Carol, I. and Prat, P. C. (1995) A multicrack model based on the theory of multisurface plasticity and two fracture energies. In Computational Plasticity (COMPLASIV), ed. D. R. J. Owen, E. Oñate and E. Hinton, Vol. 2, pp. 1583-1594. Pineridge Press, Barcelona.

Carol, I., Prat, P. C. and Gettu, R. (1993) Numerical analysis of mixed-mode fracture of quasibrittle materials using a multicrack constitutive model. In Mixed-Mode Fatigue and Fracture, 
ed. H. P. Rossmanith and K. J. Miller, pp. 319-332. Mechanical Engineering Publications Ltd., London.

Carol, I., Prat, P. C. and López, C. M. (1996) A normal/shear cracking model. Multicrack implementation for smeared analysis. Technical report, Int. Center for Numerical Methods in Engng. (CIMNE), ETSECCPB-UPC, E-08034 Barcelona, in preparation.

Christensen, R. M. and Lo, K. H. (1979) Solutions for effective shear properties in three-phase sphere and cylinder models. J. Mech. Phys. Solids 27, 315-330.

Dvorak, G. J. (1992) Transformation field analysis of inelastic composite materials. Proc. Roy. Soc. London A437, 311-327.

Dvorak, G. J. and Benveniste, Y. (1992) Transformation field analysis of inelastic composite materials. Proc. Roy. Soc. London A437, 291-310.

Eshelby, J. D. (1957) The determination of the elastic field of an ellipsoidal inclusion, and related problems. Proc. Roy. Soc. London A241, 376-396.

Fabrikant, U. I. (1987) Close interaction of coplanar circular cracks in an elastic medium. Acta Mech. 67, 39-59.

Fares, N. (1993) Effective stiffness of a periodically cracked 3-D solid. Int. J. Fracture 62, 149-162.

Hashin, Z. (1988) The differential scheme and its application to cracked materials. J. Mech. Phys. Solids 36, 719-734.

Hershey, A. V. and Dahlgren, V. A. (1954) The elasticity of an anisotropic aggregate of anisotropic cubic crystals. J. Appl. Mech. 21, 236-241.

Hill, R. (1965) A self-consistent mechanics of composite materials. J. Mech. Phys. Solids 13, 213-222.

Hoenig, A. (1978) The behavior of a flat elliptical crack in an anisotropic elastic body. Int. J. Solids and Structures 14, 925-934.

Hoenig, A. (1979) Elastic moduli of a non-randomly cracked body. Int. J. Solids Structures $15,137-154$.

Horii, H. and Nemat-Nasser, S. (1983) Overall moduli of solids with microcracks: loadinduced anisotropy. J. Mech. Phys. Solids 31, 155-171.

Horii, H. and Nemat-Nasser, S. (1985) Elastic fields of interacting inhomogeneities. Int. J. Solids Structures 21, 731-745.

Hu, K. X. and Huang, Y. (1993) Estimation of the elastic properties of fractured rock masses. Int. J. Rock Mech. Min. Sci. 30, 381-394.

Huang, Y., Hu, K. X. and Chandra, A. (1994) A self-consistent mechanics method for solids containing inclusions and a general distribution of cracks. Acta Mech. 105, 69-84.

Jirásek, M. and Bažant, Z. P. (1994) Localization analysis of nonlocal model based on crack interactions. ASCE J. Engng Mech. 120, 1521-1542.

$\mathrm{Ju}, \mathrm{J}$. W. and Chen, T.-M. (1994) Effective elastic moduli of two-dimensional brittle solids with interacting microcracks. Part I : Basic formulations. Part II : Evolutionary damage models. J. Appl. Mech. 61, 349-366.

$\mathrm{Ju}, \mathrm{J}$. W. and Tseng, K. H. (1992) A three-dimensional statistical micromechanical theory for brittle solids with interacting microcracks. Int. J. Damage Mech. 1, 102-131.

Kachanov, M. (1980) Continuum model of medium with cracks. ASCE J. Engng Mech. Div. 106(EM5), 1039-1051.

Kachanov, M. (1987a) Elastic solids with many cracks: a simple method of analysis. Int. J. Solids Structures 23, 23-43.

Kachanov, M. (1987b) On modelling of anisotropic damage in elastic-brittle materials-a brief review. In Damage Mechanics of Composites, ed. A. Wang and G. Haritos, ASME Winter, Annual Meeting, pp. 99-105. ASME, Boston.

Kachanov, M. (1992) Effective elastic properties of cracked solids : critical review of some basic concepts. Appl. Mech. Rev. 45, 304-335.

Kachanov, M. (1993) Elastic solids with many cracks and related problems. In Advances in Applied Mechanics, ed. J. Hutchinson and T. Wu, Vol. 30, pp. 259-445. Academic Press.

Kachanov, M., Tsukrov, I. and Shafiro, B. (1994) Effective moduli of solids with cavities of various shapes. Appl. Mech. Rev. 47, S151-S174.

Kröner, E. (1958) Berechnung der elastichen Konstanten des Vielkristalls aus den Konstanten des Einkristalls. Z. Physik 151, 504-518. 
Mauge, C. and Kachanov, M. (1992) Interacting arbitrarily oriented cracks in anisotropic matrix. Stress intensity factors and effective moduli. Int. J. Fracture 58, R69-R74.

Mauge, C. and Kachanov, M. (1994) Effective elastic properties of an anisotropic material with arbitrarily oriented interacting cracks. J. Mech. Phys. Solids 42, 561-584.

Mori, T. and Tanaka, K. (1973) Average stress in matrix and average elastic energy of materials with misfitting inclusions. Acta Metall. 21, 571-574.

Nemat-Nasser, S. and Yu, N. (1993) Solids with periodically distributed cracks. Int. J. Solids Structures 30, 2071-2095.

Prat, P. C. and Bažant, Z. P. (1991) Microplane model for triaxial deformation of saturated cohesive soils. ASCE J. Geotech. Engng 117, 891-912.

Roscoe, R. A. (1952) The viscosity of suspensions of rigid spheres. Brit. J. Appl. Phys. 3, 267269.

Salganik, R. L. (1973) Mechanics of bodies with many cracks. Mekhanika Tverdogo Tela (Mech. Solids) 8, 135-143.

Sayers, C. M. and Kachanov, M. (1991) A simple technique for finding effective elastic constants of cracked solids for arbitrary crack orientation statistics. Int. J. Solids Structures 27, 671-680.

Sayers, C. M. and Kachanov, M. (1995) Microcrack-induced elastic wave anisotropy of brittle rocks. J. Geophys. Res. 100(B3), 4149-4156.

Stroud, A. H. (1971) Approximate Calculation of Multiple Integrals. Prentice Hall, Englewood Cliffs, New Jersey.

Vakulenko, A. A. and Kachanov, M. (1971) Continuum theory of medium with cracks. Mech. Solids 6, 145-151.

Zimmerman, R. W. (1985) The effect of microcracks on the elastic moduli of brittle materials. J. Mater. Sci. Lett. 4, 1457-1460. 


\section{ADDENDUM (WITH ERRATA)}

\section{TANGENTIAL STIFFNESS OF ELASTIC MATERIALS WITH SYSTEMS OF GROWING OR CLOSING CRACKS}

Prat, P. C. and Bažant, Z. P. (1997) J. Mech. Phys. Solids 45, 611-636.

The purpose of this Addendum with Errata is twofold - to outline an improvement of the formulation that leads to a smaller system of equations to solve (in detail, see Bažant et al., 1997), and to correct an error. Equations (3) and (2l) of the paper did not correctly express the release of energy per unit volume of the material caused by crack growth. The first terms on the right-hand sides of these equations must be deleted because the derivatives of the complementary energy density with respect to the effective crack diameter $\vec{a}$ (or $\bar{a}_{\mu}$ ) must be taken at constant stress tensor $\sigma$. (The writers are indebted to Christian Huet, Professor at the Swiss Federal Institute of Technology, for pointing out this oversight ; also to Milan Jirásek, Research Engineer at that Institute; and Jaime Planas, Professor at the Technical University Madrid, for some other stimulating discussions.)

The expression of the strain increment tensor for the case of $N$ crack families is $\Delta \varepsilon=C: \Delta \sigma+\sum_{\mu}\left(\bar{c} C \hat{c} \bar{a}_{\mu}\right): \sigma \Delta \bar{a}_{\mu}$. This may be inverted as

$$
\Delta \sigma=E:\left(\Delta \varepsilon-\sum_{\mu=1}^{*} \frac{\hat{c} C}{\hat{\partial} \vec{a}_{\mu}}: \sigma \Delta \bar{a}_{\mu}\right)
$$

where $E$ is the fourth-order secant stiffness tensor whose $6 \times 6$ matrix is the inverse of the matrix of the secant compliance tensor $C\left(\bar{a}_{1}, \bar{a}_{N}\right)$. The energy balance equations (21) and (22) of the paper, as well as (3) for $N=1$, should be simplified as:

$$
(1 / 2) \sigma:\left(\hat{c} C: \hat{c} \bar{a}_{\mu}\right): \sigma=2 \pi n_{\mu} \kappa_{\mu} G_{f} \bar{a}_{\mu}
$$

where $\bar{a}_{\mu}$ is the effective diameter of the cracks in family $\mu$, and $\kappa_{\mu}$ is a crack growth indicator such that $\kappa_{\mu}=1$ for $\Delta \bar{a}_{\mu}>0 ; 0 \leqslant \kappa_{\mu} \leqslant 1$ for $\Delta \bar{a}_{\mu}=0$; and $\kappa_{\mu}=0$ for $\Delta \bar{a}_{\mu}<0$. Because of this change, the term involving $\Delta \sigma$ must be deleted from (5), (22) and (31). Furthermore, (4) and (6) are no longer needed and should be deleted. All the other equations remain unaffected by this change.

From (2), the incremental energy balance condition ( for $\mu=1,2, \ldots, N$ ) is :

$$
\sigma: \frac{\partial C}{\partial \bar{a}_{\mu}}: \Delta \sigma+\sum_{\nu=1}^{v}\left(\frac{1}{2} \sigma: \frac{\partial^{2} C}{\partial \bar{a}_{\mu} \partial \bar{a}_{v}}: \sigma-2 \pi n_{\mu} \kappa_{\mu} G_{j} \dot{\delta}_{\mu \nu}\right) \Delta \bar{a}_{\nu}=2 \pi n_{\mu} \bar{a}_{\mu} G_{f} \Delta \kappa_{\mu}
$$

where $\Delta \kappa_{\mu}=0$ except when the crack growth changes to crack closing. The handling of the large jump in $\kappa_{\mu}$ is exact if $\kappa_{\mu}=\kappa_{\mu}^{\text {new }}$ and $\bar{a}_{\mu}=\bar{a}_{\mu}^{\text {old }}$ (because $\left.\Delta\left(\kappa_{\mu} \bar{a}_{\mu}\right)=\left(\kappa_{\mu} \bar{a}_{\mu}\right)^{\text {ncw }}-\left(\kappa_{\mu} \bar{a}_{\mu}\right)^{\text {old }}=\kappa_{\mu}^{\text {new }} \Delta \bar{a}_{\mu}+\Delta \kappa_{\mu} \bar{a}_{\mu}^{\text {old }}\right)$. Substitution of (1) into (3) leads to a system of $N$ equations for $N$ unknowns $\Delta \bar{a}_{1}, \ldots, \Delta \bar{a}_{v}$ : 


$$
\begin{gathered}
\text { Addendum } \\
\sum_{v=1}^{N} A_{\mu \nu} \Delta \bar{a}_{v}=B_{\mu} \quad(\mu=1, \ldots, N)
\end{gathered}
$$

where

$$
\begin{gathered}
A_{\mu v}=\sigma:\left(\frac{\partial C}{\bar{c} \bar{a}_{\mu}}: E: \frac{\bar{c} C}{\partial \bar{a}_{v}}-\frac{1}{2} \frac{\partial^{2} C}{\partial \bar{a}_{\mu} \partial \bar{a}_{v}}\right): \sigma+2 \pi G_{f} n_{\mu} \kappa_{\mu}^{\text {new }} \delta_{\mu v} \\
B_{\mu}=\sigma: \frac{\partial C}{\partial \bar{a}_{\mu}}: E: \Delta \varepsilon-2 \pi n_{\mu} \bar{a}_{\mu}^{\text {old }} G_{f} \Delta \kappa_{\mu}
\end{gathered}
$$

These equations need to be further modified by introducing a check for compression. The reason is that the energy expression is quadratic, which means that (2) is invariant when $\sigma$ is replaced by $-\sigma$. Thus. negative stress intensity factor $K_{t \mu}$ can occur even when (2) is satisfied, and so the sign of $K_{l \mu}$ must be checked for each crack family $\mu$. The case $K_{\mu \mu}<0$ is inadmissible.

Since the present formulation yields only the values of $\left(K_{l_{\mu}}\right)^{2}$ but not $K_{I_{\mu}}$, the sign of $K_{l a}$ must be inferred approximately. It can be considered the same as the sign of the stress $\sigma_{v_{\mu}}=v_{\mu} \cdot \sigma \cdot v_{\mu}$ in the direction normal to the cracks of $\mu$ th family ( $v_{\mu}=$ unit vector normal to the cracks). Alternatively, the sign of $K_{t \mu}$ can be inferred from the sign of the normal component $\varepsilon_{v_{\mu}}^{c r}=v_{\mu} \cdot \varepsilon_{v}^{c r} \cdot v_{\mu}$ of the cracking strain tensor $\varepsilon^{c r}=C^{c r}: \sigma$ where $C^{\prime r}$ is given by (28). Although such estimation of the sign of $K_{I \mu}$ is only approximate for the microscale, it is consistent with the macroscopic approximation of $C$ because all the composite material models for cracked solids are based on the solution of one crack in an infinite solid. for which the sign of $K_{t, k}$ coincides with the sign of $\sigma_{v i}\left(\right.$ or $\left.\varepsilon_{v i \mu}^{c r}\right)$.

Usually the six independent components of $\Delta \varepsilon$ are known or assumed, and then (4) represents a separate system of only $V$ equations for $\Delta \bar{a}_{1}, \ldots, \Delta \bar{a}_{v}$ (this is a simplification compared to the formulation in the paper, which led to a system of $N+6$ equations). In each iteration of each loading step, the values or $\kappa_{\mu}$ are set according to the sign of $\Delta \bar{a}_{\text {, }}$ in the preceding step or preceding iteration. If $\Delta \bar{a}_{\mu}=0$, or if (due to numerical erior) $\left|\Delta \bar{a}_{\mu}\right|$ is non-zero but less than a certain chosen very small positive number $\dot{\delta}$, one may arbitrarily set $\kappa_{\mu}=0.5$.

After solving (4), evaluating $\Delta \sigma$ from (1) and incrementing $\sigma$, one must check whether the case $\Delta \vec{u}_{\mu}>0$ and $\sigma_{v_{\mu}}<0$ (or $\varepsilon_{v_{\mu}}^{c r}<0$ ) occurs for any $\mu$. If it does, the corresponding equation in the system. (4) must be replaced by the equation $\Delta \bar{a}_{\mu}=0$. The solution of such a modified equation system must be iterated until the case $\Delta \bar{a}_{\mu}>0$ and $\sigma_{v_{\mu}}<0$ (or $\varepsilon_{v \mu \mu}^{c r}<0$ ) would no longer occur for any $\mu$.

With the aforementioned refinements and corrections, the recalculated graphs are not qualitatively different from those in the original paper (Figs 8-14). Because only a qualitative agreement with the behavior of concrete was claimed in the paper, the revised graphs need not be reproduced in this Addendum.

All the conclusions stated in the paper remain valid.

\section{REFERENCE}

Bażant. Z. P., Jirásek, M. and Prat, P. C. (1997) Tangential stiffness tensor of elastic material with growing or closing cracks. Report No. 97-5/402t, Dept. of Civil Engrg, Northwestern University, Evanston, Illinois; also Mechanics Research Communications, submitted. 